\title{
A high-order finite-volume method for atmospheric flows on unstructured grids
}

\author{
Panagiotis Tsoutsanis $1 *$ and Dimitris Drikakis ${ }^{2}$ \\ ${ }^{1}$ Cranfield University, Cranfield MK43 OAL, United Kingdom and \\ ${ }^{2}$ University of Strathclyde, Glasgow G1 1XJ, United Kingdom
}

(Dated: October 26, 2016)

\begin{abstract}
This paper presents an extension of a Weighted Essentially Non-Oscillatory (WENO) type schemes for the compressible Euler equations on unstructured meshes for stratified atmospheric flows. The schemes could be extended for regional and global climate models dynamical cores. Their potential lies in their simplicity; accuracy; robustness; non-oscillatory properties; versatility in handling any type of grid topology; computational and parallel efficiency. Their defining characteristic is a non-linear combination of a series of high-order reconstruction polynomials arising from a series of reconstruction stencils. In the present study an explicit Strong Stability Preserving (SSP) Runge-Kutta $3^{\text {rd }}$-order method is employed for time advancement. The WENO schemes (up to $5^{\text {th }}$-order) are applied to the two dimensional and three dimensional test cases: a 2D rising thermal bubble; the 2D density current and the 3D Robert smooth bubble. The parallel performance of the schemes in terms of scalability and efficiency is also assessed.
\end{abstract}

Keywords:Unstructured Mesh, Finite-Volume, WENO, Stratified Atmospheric Flows.

PACS: 89.75.-k, 89.75.Da, 05.45.Xt, 87.18.-h

MSC: 34C15, 35Q70

*Corresponding author. Email: panagiotis.tsoutsanis@cranfield.ac.uk, Tel.: +44 1234 754635, Fax: +44 1234758207.

${ }^{\dagger}$ Email: dimitris.drikakis@strath.ac.uk 
Journal of Coupled Systems and Multiscale Dynamics • October 2016

\section{INTRODUCTION}

Understanding the atmospheric processes both qualitatively and quantitatively on regional and global scales is of great value for scientists and policy decision makers. Tools utilised by the scientific community to gain insight into the complex atmosphere-climate system, include the numerical weather prediction (NWP) and climate models. The main objective is to accurately simulate weather and climate variations, trends, patterns etc. All the models utilised for this purpose have a distinctive component in common, the dynamical core, which solves the fluid dynamics equations. In other words it is the model component that drives atmospheric motions; hence with a profound impact on the accuracy of the predictions. The leap occurring in computing processing power the last decade, provides a motivation to resolve atmospheric processes on finer scales and higher resolution than happened before. There is currently a trend of either enhancing the majority of the dynamical cores of established models or develop new dynamical cores that employ highly sophisticated algorithms or set of equations needed for the next generation of high performance computing facilities. A number of initiatives have been taken to address this bottleneck and develop the next generation of dynamical cores. Such initiatives include the UK Met Office and European Centre for Medium-Range Weather Forecasts Even Newer Dynamics for General Atmospheric Modelling of the Environment (ENDGame) [55]; the National Center for Atmospheric Research High-Order Methods Modelling Environment [36]; the National Oceanic and Atmospheric Administration AM3 model [37] and the Nonhydrostatic Icosahedral Model (NIM) [38]; the Frontier Research Center for Global Change Nonhydrostatic Icosahedral Atmospheric Model, (NICAM) [43]; the Non-hydrostatic Unified Model of the Atmosphere (NUMA) [18]; and the Max Planck Institute for Meteorology and Deutscher Wetterdienst ICOsahedral Non-hydrostatic general circulation models, (ICON) [33].

This paper focuses on the numerical methods component of the dynamical core aspect of atmo-

spheric flows. Since high performance computing systems enable the analysis on finer scales and higher resolution, the topography should be efficiently and accurately represented, a property typical for unstructured grids. In the past, finite volume numerical methods have been introduced in the structured grid context resulting in robust schemes that enjoyed a wide recognition in various disciplines, including the weather and climate prediction community, e.g. [28, 29, 47].

The first generation numerical methods for unstructured grids also suffered from low accuracy compared to structured grids and their requirements in terms of computing power and resources were 
higher. However, the numerical methods for unstructured grids have matured, and numerous elegant approaches [10, 13, 19, 22, 30, 39, 41, 50, 52, 54, 60-62] and algorithms have been developed in the finite volume framework for a wide range of applications for Computational Fluid Dynamics(CFD). Other state-of-the-art approaches are also available, such as the Discontinuous Galerkin (DG) [7, 8, 10, 56, 61, 68], and Spectral Finite-Volume (SFV) methods [6, 57-59, 62, 67] that have been successfully applied for CFD applications. For the finite volume framework the first class of high-resolution methods developed for unstructured grids included the ENO type schemes [1, 48], followed by the WENO type schemes [14, 24, 44, 45]. In the WENO case, the high-order accuracy was achieved by non-linearly combining a series of high-order reconstruction polynomials arising from a series of reconstruction stencils. Recently, a class of WENO type methods [50, 54] has been successfully extended to hybrid unstructured meshes with various geometrical shapes such as tetrahedrals, hexahedrals, prisms, and pyramids . It has been demonstrated that they can achieve the very high order of spatial accuracy across interfaces between cells of different types, and at the same time essentially non-oscillatory profiles are produced for discontinuous solutions. This gives greater flexibility to handle complex geometrical shapes in an efficient and accurate manner. The approximations used in the subject schemes that dictate their performance apart from the governing equations employed are related to the order of approximation of the surface, and volume integrals by a Gaussian quadrature rule of suitable order for the order of polynomials utilised. Additionally the initial condition for any flow problem with space dependent solution vary with various orders of accuracy, since the Gaussian quadrature points and weights used are also dependent on the spatial scheme chosen. It has beed demonstrated in a previous study [53] that the chosen variables used for reconstruction have a profound impact on the performance of the subject schemes at low-Mach number flow problems, where the characteristic variable based reconstructions do not exhibit the same accuracy problem as conserved variables for low-Mach number flows using the compressible flow equations.

For the atmospheric dynamics there is also an overwhelming number of recent state-of-the-art approaches that utilise high-order schemes for either structured grid or unstructured dynamical cores [5, 8, 15, 16, 25, 31, 34, 46, 63-65]. Regarding the high-order schemes (higher than 3rd order of spatial accuracy) for unstructured dynamical cores all of the approaches are based on the Discontinuous Galerkin framework and the Spectral Elements Method (SE) [8, 16, 27, 34, 64]. One of the primary aims of the next generation of NWP and climate prediction dynamical cores is to resolve phenomena of finer scales, by taking into account non-hydrostatic processes. Therefore there is a need for adopting or 
developing better-suited formulations of the governing equations, and matching them with the appropriate numerical methods. To the best of our knowledge there are no approaches that use WENO-class of higher-order schemes for unstructured meshes in the finite volume framework for atmospheric flows. The fact that WENO schemes have been successfully applied previously for flow problems including smooth and discontinuous flow regions [3, 4, 9, 10, 13, 52], motivates their extension for atmospheric dynamics problems.

The aim of this paper is to apply and assess the performance and suitability of WENO-class of higher-order finite volume schemes for unstructured meshes for well established test cases for atmospheric dynamics. It must be stressed that these schemes are independent of element shapes, and are generic in the sense that they can be employed for any type of meshes.

The paper is organised as follows. In Section 2 the governing equations of a dry stratified atmosphere are outlined. Section 3 presents a detailed explanation of the spatial discretisation techniques in terms of stencil construction algorithms, WENO reconstruction, numerical fluxes, source terms and temporal discretisation. The results obtained with various schemes for different test cases, including the parallel performance are presented in Section 4. Section 5 summarises the conclusions drawn from the present study.

\section{GOVERNING EQUATIONS}

The compressible Euler equations employed in the CFD community where mass, momentum and total energy are the conserved quantities are rarely used for atmospheric studies, with a few notable exceptions [17]. The primary reason for not being that common in atmospheric studies is the additional computational step required to compute potential temperature from total energy. However the benefits of using these set of equations are their formally conservative nature as reported in [17], and the fact that existing state-of-the-art methods and techniques already developed in the CFD community can be readily applied with minor modifications if required to model atmospheric processes. The compressible non-hydrostatic Euler equations without moisture effects are considered in the following form:

$$
\frac{\partial}{\partial t} \mathbf{U}+\frac{\partial}{\partial x} \mathbf{F}(\mathbf{U})+\frac{\partial}{\partial y} \mathbf{G}(\mathbf{U})+\frac{\partial}{\partial z} \mathbf{H}(\mathbf{U})=\mathbf{S},
$$

where $\mathbf{U}$ is the vector of the conserved variables, $\mathbf{F}, \mathbf{G}, \mathbf{H}$ are the flux vectors in $x, y$ and $z$ directions of Cartesian coordinates and $\mathbf{S}$ is a gravity source function given by 


$$
\begin{gathered}
\mathbf{U}=\left[\begin{array}{c}
\rho \\
\rho u \\
\rho v \\
\rho w \\
E
\end{array}\right], \mathbf{F}=\left[\begin{array}{c}
\rho u \\
\rho u^{2}+p \\
\rho u v \\
\rho u w \\
u(E+p)
\end{array}\right] \\
\mathbf{H}=\left[\begin{array}{c}
\rho w \\
\rho w u \\
\rho w v \\
\rho w^{2}+p \\
w(E+p)
\end{array}\right], \mathbf{G}=\left[\begin{array}{c}
\rho v \\
\rho v u \\
\rho v^{2}+p \\
\rho v w \\
v(E+p)
\end{array}\right], \\
\mathbf{S}=\left[\begin{array}{c}
0 \\
0 \\
0 \\
-\rho g \\
0
\end{array}\right]
\end{gathered}
$$

where $\rho$ is density; $u, v, w$ are the velocity components in the $x, y$ and $z$ directions of Cartesian coordinates, respectively; $p$ is pressure; $E=\rho c_{v} T+(1 / 2) \rho\left(u^{2}+v^{2}+w^{2}\right)+\rho g y$ is the total energy per unit mass; $\gamma$ is the ratio of specific heats; $g$ the gravitational constant; $g y$ is the geopotential height; $T$ is the temperature; $R=c_{p}-c_{v}$ is the gas constant, where $c_{p}$ is the specific heat at constant pressure and $c_{v}$ is the specific heat at constant volume. The ideal gas law is used with $\gamma=1.4$ throughout this work, with the pressure $p$ given by

$$
p=p_{s}\left(\frac{\rho R \theta}{p_{s}}\right)^{\gamma},
$$

where $p_{s}$ is the atmospheric pressure at sea level, and $\theta$ is the potential temperature

$$
\theta=\frac{T}{\pi}
$$

with $\pi$ being the Exner pressure provided by the following expression

$$
\pi=\left(\frac{p}{p_{s}}\right)^{\frac{R}{c_{p}}}
$$


The following equations relate the Exner pressure $\pi$ to the density $\rho$, and the potential temperature $\theta$ to the total energy per unit mass $E$ :

$$
\rho=\frac{p_{s}}{R \theta} \pi^{\frac{c_{v}}{R}}
$$

and

$$
E=\rho c_{v} \theta \pi+(1 / 2) \rho\left(u^{2}+v^{2}+w^{2}\right)+\rho g y .
$$

The Exner pressure and potential temperature can be split in their mean and perturbed parts as follows

$$
\theta(x, y, z, t)=\bar{\theta}(y, t)+\theta^{\prime}(x, y, z, t)
$$

and

$$
\pi(x, y, z, t)=\bar{\pi}(y, t)+\pi^{\prime}(x, y, z, t)
$$

where the hydrostatic balance of their mean values is given by

$$
c_{p} \bar{\theta} \frac{d \bar{\pi}}{d y}=-g .
$$

Integrating in space over a mesh element $V_{i}$, we obtain the following semi-discrete finite-volume method

$$
\frac{\partial \mathbf{U}_{i}}{\partial t}+\frac{1}{\left|V_{i}\right|} \oint_{\partial V_{i}} \mathbf{F}_{n} d A=\mathbf{S}_{\mathbf{i}},
$$

and using the rotational invariance property of the Euler equations [51]

$$
\begin{aligned}
\mathbf{F}_{n}(\mathbf{U}) & =\mathbf{F}(\mathbf{U}) n_{x}+\mathbf{G}(\mathbf{U}) n_{y}+\mathbf{H}(\mathbf{U}) n_{z} \\
& =\mathbf{T}^{-\mathbf{1}} \mathbf{F}(\mathbf{T} \mathbf{U}),
\end{aligned}
$$

where $\mathbf{n}=\left(n_{x}, n_{y}, n_{z}\right)$ is the outward unit normal vector; $\mathbf{U}_{i}(t)$ are the cell averages of the solution at time $t ; \mathbf{F}_{n}$ is the projection of the flux tensor on the normal direction; $\mathbf{T}$ is the rotation matrix; and $\mathbf{T}^{-1}$ its inverse [51]; and the source term $\mathrm{S}_{\mathbf{i}}$ is given by 


$$
\mathbf{S}_{\mathbf{i}}=\frac{1}{\left|V_{i}\right|} \int_{V_{i}} S(x, y, z, t) d x d y d z,
$$

Assuming that the element's surface consists of $L$ faces, with the spatial index $i$ being omitted for simplicity,

$$
\partial V_{i}=\sum_{j}^{L} A_{j},
$$

( $\mathbf{n}_{j}$ denoting the outward unit vector for face $A_{j}$ ), then the integral over the element boundary $\partial V_{i}$ can be split into the sum of integrals over each face, resulting in the following expression:

$$
\frac{\partial \mathbf{U}_{i}}{\partial t}=\mathbf{R}_{i}
$$

where

$$
\begin{aligned}
\mathbf{R}_{i} & =-\frac{1}{\left|V_{i}\right|} \sum_{j=1}^{L} \int_{A_{j}} \mathbf{F}_{n, j} d A+\mathbf{S}_{\mathbf{i}} \\
& =-\frac{1}{\left|V_{i}\right|} \sum_{j=1}^{L} \mathbf{K}_{i j}+\mathbf{S}_{\mathbf{i}} .
\end{aligned}
$$

The numerical flux $\mathbf{K}_{i j}$ corresponding to face $j$ of the cell $V_{i}$ is the surface integral of the projection of the tensor of fluxes onto $\mathbf{n}_{j}$. In a numerical method the exact integral expression for the numerical flux $\mathbf{K}_{i j}$ for the face $j$ of a cell $V_{i}$ is approximated by a suitable Gaussian numerical quadrature

$$
\mathbf{K}_{i j}=\int_{A_{j}} \mathbf{F}_{n, j} d A=\sum_{\beta} \mathbf{F}_{n, j}\left(\mathbf{U}\left(\mathbf{x}_{\beta}, t\right)\right) \omega_{\beta}\left|A_{j}\right|,
$$

where the subscript $\beta$ corresponds to different Gaussian integration points $\mathbf{x}_{\beta}$ and weights $\omega_{\beta}$ over the face $A_{j}$.

\section{NUMERICAL FRAMEWORK}

In this section the numerical framework for the solution of the compressible non-hydrostatic Euler equations is presented with respect to spatial and temporal discretisation. 


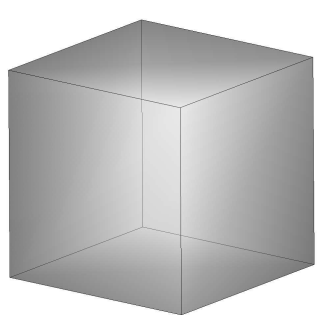

(a) Hexahedral

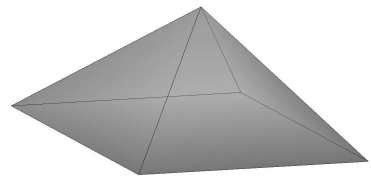

(b) Pyramid

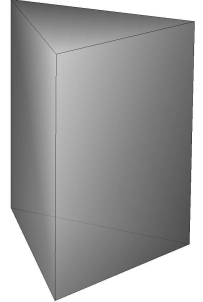

(c) Prism

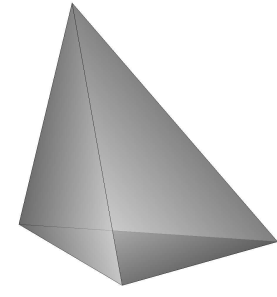

(d) Tetrahedral

FIG. 1: 3D Element shapes

\subsection{Spatial discretisation}

The spatial discretisation is based on the approach of [50, 54] which is suitable for unstructured meshes with various types of element shapes in 2D and 3D, where it has been previously used successfully for laminar, transitional and turbulent flows [52], and only the key characteristics of this approach are going to be described in this paper. The first step in the approximation of the governing equations concerns the spatial discretisation of the domain $\Omega \subset R^{3}$ into $E$ number of conforming elements $V_{i}$, with the index $i$ ranging from 1 to $E$ number of elements. The elements can be any combination of hexahedrals, pyramids, prisms, and tetrahedrals as shown in Fig. 11in 3D, and triangles or quadrilaterals in $2 \mathrm{D}$. It must be noted that the present WENO schemes are not limited by conforming meshes, since they can be used with non-conforming meshes which brings additional benefits such as adaptive mesh refinement [11].

The combination of all elements in the spatial domain is given by

$$
\Omega=\bigcup_{i=1}^{E} V_{i} .
$$

In the context of the finite volume framework that is employed in the present study, the data is represented by cell averages of conserved variable $U(x, y, z)$ in each element $V_{i}$

$$
\bar{U}_{i}=\frac{1}{\left|V_{i}\right|} \int_{V_{i}} U(x, y, z) d V,
$$

where $\left|V_{i}\right|$ is the volume of the element. To achieve high-order, accurate spatial discretisation, highorder accurate point-wise values of the solution need to be recovered from the cell averages. The 
reconstruction problem can thus be reformulated as follows: For a target cell $V_{0}$ we build a high-order polynomial $p_{i}(x, y, z)$ that has the same cell average as $U$ on the target cell

$$
\begin{aligned}
\bar{U}_{i} & =\frac{1}{\left|V_{i}\right|} \int_{V_{i}} U(x, y, z) d V \\
& =\frac{1}{\left|V_{i}\right|} \int_{V_{i}} p_{i}(x, y, z) d V .
\end{aligned}
$$

The point-wise values of the conserved variable $U$ in each cell are approximated with a polynomial of a desired order of accuracy $r$. The polynomial uses the cell averages of $U(x, y, z)$ on the target cell $V_{i}$ as well as averages $\bar{U}_{m}$ from neighbouring cells, $V_{m}$.

\subsubsection{Stencil selection algorithm}

For high-order finite-volume methods the variation of information (data) in the close spatial proximity (neighbourhood) of each element in the mesh is required. Therefore, a high quality approximation of how this information varies within each element must be obtained. Hence, it is required to construct a region (neighbourhood) of elements surrounding each element in the mesh. This region is named the stencil and is constructed by recursively adding the direct side neighbours of any considered element until a desired number of elements has been reached. The basic steps of the stencil construction procedure are as follows:

1. For cell $i$ we want to construct a set of elements $S$ (stencil) consisting of $N(S)$ elements

2. With $c=1,2, \ldots . N$ being the index of the numbering of the elements in the stencil

3. $S_{1}=i$, the considered cell $i$ is always the first element in the stencil $c=1$

4. Assume that the element in the stencil $S_{c}$ has $M$ number of direct neighbours

(a) Check which of the M elements do not belong in the set (repetition condition)

(b) If WENO scheme is selected check which of the resulting M elements that do not belong in the set satisfy the geometrical sector conditions (geometrical conditions)

5. Assign as the next elements in the stencil only the ones that do not belong in the set

6. Repeat steps 4 to 5 until $N$ number of elements have been assigned 


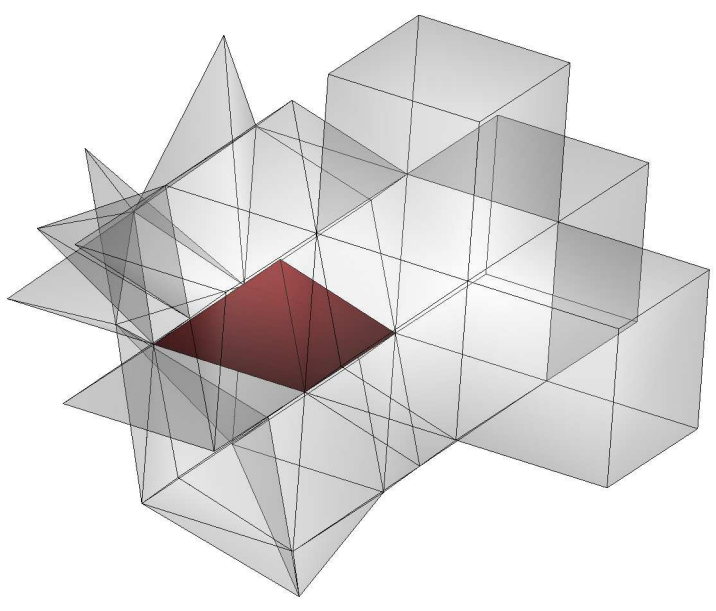

(a) Hybrid stencil

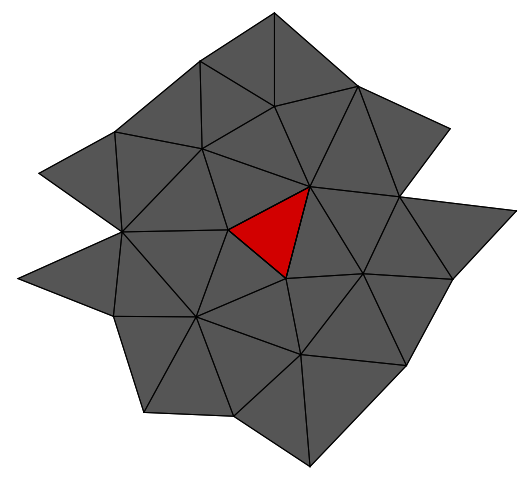

(b) Triangular stencil

FIG. 2: Central stencils with the considered cell in red colour (colour online).

Typical examples of central stencils in three-dimensions can be seen in Fig. 2, where the considered cell $i$ is illustrated in red colour. There is another category of stencils named the directional stencils or WENO stencils subject to an additional condition. The additional condition that must be satisfied is that the candidate element must lie within a specified geometrical sector. Specifically for the subject study the cell centers of the candidate elements must lie within the geometrical sectors defined by the cell centre of the considered cell and the vertices of each of the faces/edges. Therefore the number of directional stencils is equal to the number of faces/edges as seen in Fig. 4. The same approach as [50, 54] is utilised for the definition of geometrical sectors. Typical examples of directional stencils in three-dimensions can be seen in Fig. 3, where the considered cell $i$ is illustrated in red colour.

\subsubsection{Reconstruction algorithm}

One crucial requirement for the reconstruction process for unstructured meshes is that the algorithm should be as mesh independent as possible, since bad-quality elements should not contaminate the solution, and therefore the basis functions used should not be restricted to one ideal element type. Since relying on the transformation from physical to computational space and vice-versa may not be achievable for highly distorted hexahedral,prismatic and pyramidal elements. This requirement poses 


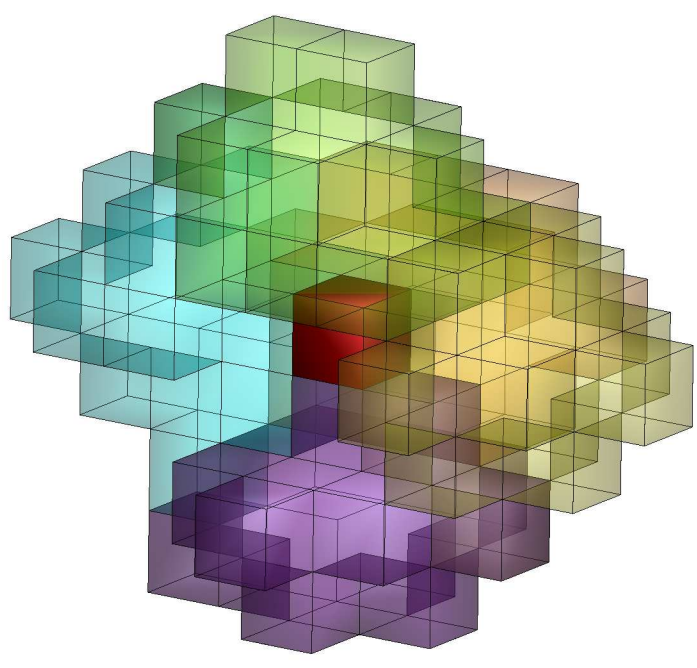

(a) Hexahedral directional stencils

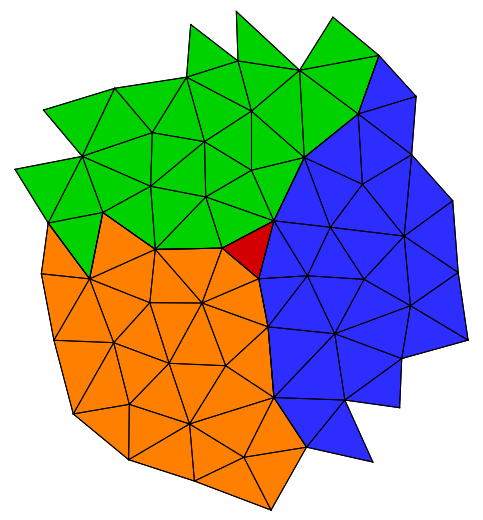

(b) triangular directional stencils

FIG. 3: Directional stencils depicted by different colours (colour online).

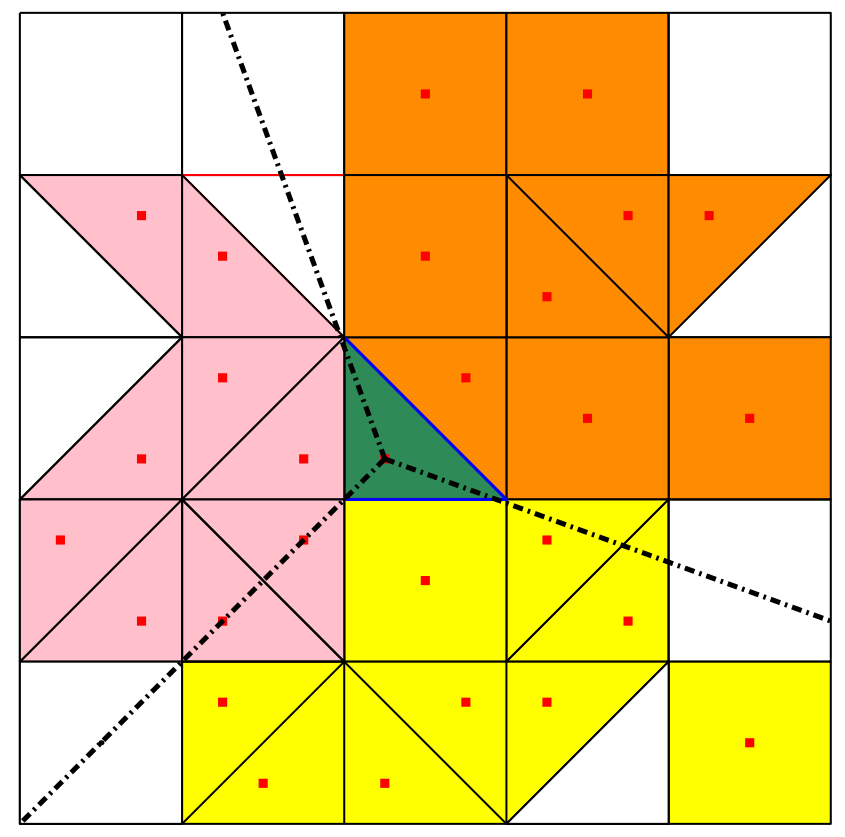

(a) Example 1

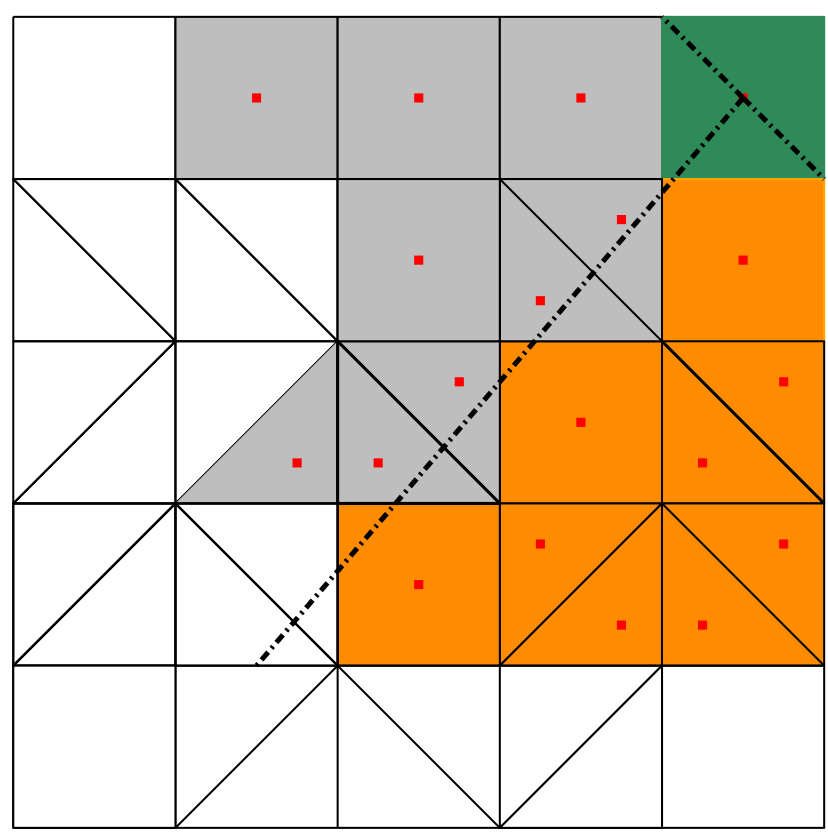

(b) Example 2

FIG. 4: Schematic representation of the directional stencils sectors for a WENO 3rd-order scheme on a 2D mesh, with the considered cell in green colour, the geometrical sectors defined by dotted lines, and the cell centres in red colour (colour online). 
a lot of challenges since it is quite common to encounter distorted elements in unstructured meshes for complicated geometries, which can contain non-planar faces. Therefore the reconstruction algorithm should be able to handle these types of elements, by decomposing them in tetrahedral elements where the transformation from physical to reference space is always achieved. The main steps for the reconstruction process are the following:

1. If the considered cell $i$ is not a tetrahedral and contains non-planar quadrilateral faces (such as hexahedral, prisms and pyramids) then decompose it into tetrahedral elements. It must be stressed that the decomposition to tetrahedral cells, is done only for the transformation purposes to the reference space in order to remove any scaling effect of the least square system to be solved.

2. Choose one of the resulting decomposed elements.

3. Transform the chosen decomposed element from the physical space described by the Cartesian coordinates $x, y, z$ into a reference space described by $\xi, \eta, \zeta$.

4. Based on the Jacobian matrix of the transformation of the chosen decomposed element, map the coordinates of the entire element into the reference space described by coordinates $\xi, \eta, \zeta$.

5. Based on the same Jacobian, all the elements in the stencil are transformed to the reference space and their volumes, and barycentres positions are recomputed in the new reference space.

Let $\mathbf{v}_{i j}, j=1,2, \ldots J_{i}$ be the vertices of the considered (general) element, which can be either tetrahedral, hexahedral, prismatic or pyramidal. By decomposing the element into tetrahedrals and choosing one of them with $\mathbf{w}_{1}=\left(x_{1}, y_{1}, z_{1}\right), \mathbf{w}_{2}=\left(x_{2}, y_{2}, z_{2}\right), \mathbf{w}_{3}=\left(x_{3}, y_{3}, z_{3}\right), \mathbf{w}_{4}=\left(x_{4}, y_{4}, z_{4}\right)$ being it's four vertices. Obviously, these vertices are between the $\mathbf{v}_{i j}$ ones. The transformation from the Cartesian coordinates $x, y, z$ into a reference space $\xi, \eta, \zeta$ is given by the following equations

$$
\left(\begin{array}{l}
x \\
y \\
z
\end{array}\right)=\left(\begin{array}{l}
x_{1} \\
y_{1} \\
z_{1}
\end{array}\right)+J \cdot\left(\begin{array}{l}
\xi \\
\eta \\
\zeta
\end{array}\right),
$$

with the Jacobian matrix given by

$$
J=\left[\begin{array}{ccc}
x_{2}-x_{1} & x_{3}-x_{1} & x_{4}-x_{1} \\
y_{2}-y_{1} & y_{3}-y_{1} & y_{4}-y_{1} \\
z_{2}-z_{1} & z_{3}-z_{1} & z_{4}-z_{1}
\end{array}\right] .
$$


Using an inverse mapping the element $V_{i}$ can be transformed to the element $V_{i}^{\prime}$ in the reference co-ordinate system

$$
\mathbf{v}_{i j}^{\prime}=J^{-1} \cdot\left(\mathbf{v}_{i j}-\mathbf{w}_{1}\right), \quad j=1,2, \ldots J_{i} .
$$

The spatial average of $U(x, y, z)$ does not change during transformation

$$
\begin{gathered}
\bar{U}_{i}=\frac{1}{\left|V_{i}\right|} \int_{V_{i}} U(x, y, z) d V \equiv \\
\frac{1}{\left|V_{i}^{\prime}\right|} \int_{V_{i}^{\prime}} U(\xi, \eta, \zeta) d \xi d \eta d \zeta .
\end{gathered}
$$

To perform the reconstruction on the target element $V_{i}$, we form the central reconstruction stencil $\mathcal{S}$ as described previously, consisting of $M+1$ elements, including the target element $V_{i}$

$$
\mathcal{S}=\bigcup_{m=0}^{M} V_{m},
$$

where the index $m$ refers to the local numbering of the elements in the stencil, with the element with index 0 being the considered cell $i$. The $r^{\text {th }}$ order reconstruction polynomial at the transformed cell $V_{0}^{\prime}$ is sought as an expansion over local polynomial basis functions $\phi_{k}(\xi, \eta, \zeta)$ :

$$
\begin{aligned}
p(\xi, \eta, \zeta) & =\sum_{k=0}^{K} a_{k} \phi_{k}(\xi, \eta, \zeta) \\
& =\bar{U}_{0}+\sum_{k=1}^{K} a_{k} \phi_{k}(\xi, \eta, \zeta),
\end{aligned}
$$

where $a_{k}$ are degrees of freedom and the upper index in the summation of expansion $K$ is related to the order of the polynomial $r$ by the expression $K=\frac{1}{6}(r+1)(r+2)(r+3)-1$ for 3D and $K=\frac{1}{2}(r+1)(r+2)-1$ for $2 \mathrm{D}$. The conservation condition (17) imposes an important constraint on the basis functions: they must have zero mean value over the cell $V_{0}^{\prime}$. On purely tetrahedral meshes hierarchical orthogonal reconstruction basis functions [13, 61], defined on the reference element, satisfy this requirement automatically. Since our general cells are not necessarily transformed onto a unit tetrahedron or cube, we need to construct basis functions $\phi_{k}$ in such a way that the condition is satisfied identically, irrespective of the degrees of freedom. The basis functions are defined as follows: 


$$
\begin{gathered}
\phi_{k}(\xi, \eta, \zeta) \equiv \psi_{k}(\xi, \eta, \zeta)-\frac{1}{\left|V_{0}^{\prime}\right|} \int_{V_{0}^{\prime}} \psi_{k} d \xi d \eta d \zeta, \\
k=1,2, \ldots
\end{gathered}
$$

Any type of orthogonal polynomial basis function can be utilised, while in the present study generic polynomial basis functions are used

$$
\left\{\psi_{k}\right\}=\xi, \eta, \zeta, \xi^{2}, \eta^{2}, \zeta^{2}, \xi \cdot \eta, \xi \cdot \zeta, \zeta \cdot \eta, \xi \cdot \eta \cdot \zeta \ldots
$$

To find the unknown degrees of freedom $a_{k}$ for each cell $V_{m}^{\prime}$ from the stencil the cell average of the reconstruction polynomial $p(\xi, \eta, \zeta)$ must be equal to the cell average of the solution $\bar{U}_{m}$ :

$$
\begin{aligned}
\int_{E_{m}^{\prime}} p(\xi, \eta, \zeta) d \xi d \eta d \zeta & =\left|V_{m}^{\prime}\right| \bar{U}_{0}+\sum_{k=1}^{K} \int_{V_{m}^{\prime}} a_{k} \phi_{k} d \xi d \eta d \zeta \\
& =\left|V_{m}^{\prime}\right| U_{m}, \quad m=1, \ldots
\end{aligned}
$$

Denoting the integrals of the basis function $k$ over the cell $m$ in the stencil, the vector of right-hand side by $A_{m k}$ and $\mathbf{b}$ are given by

$$
A_{m k}=\int_{V_{m}^{\prime}} \phi_{k} d \xi d \eta d \zeta, \quad b_{m}=\left|V_{m}^{\prime}\right|\left(\bar{U}_{m}-\bar{U}_{0}\right)
$$

We can rewrite the equations for degrees of freedom $a_{k}$ in a matrix form as

$$
\sum_{k=1}^{K} A_{m k} a_{k}=b_{m}, \quad m=1,2, \ldots M .
$$

The three-dimensional integrals on the left-hand side of (28) are calculated using Gaussian quadratures of appropriate order. In general, to compute the degrees of freedom $a_{k}$ we need at least $K$ cells in the stencil, different from the target cell $E_{0}$. However, the use of the minimum possible number of cells in the stencil $M \equiv K$ results in a scheme which may become unstable on general meshes $[12,22,23,54]$. It is, therefore, recommended to use more cells in the stencil than the minimum required number. Although it is usually sufficient to use 50\% more cells, for mixed-element meshes it is safer to increase the stencil further. We typically select $M=2 \cdot K$.

Since the resulting system becomes over-determined, the least-square procedure is invoked to solve it. The least-square reconstruction is obtained by seeking the minimum of the following functional 


$$
\mathcal{F}=\sum_{m=1}^{M} w_{m} \cdot\left(\sum_{k=1}^{K} A_{m k} a_{k}-b_{m}\right)^{2},
$$

where the weights $w_{m}$ are squared reciprocals of the distance between cells $E_{0}^{\prime}$ and $E_{m}$. The advantage of the weighted least square reconstruction is that the influence of the data farther from the considered $E_{0}^{\prime}$ is reduced, although a central least square reconstruction is materialised with the weights $\omega_{m}$ being equal to unity. Minimisation of $\mathcal{F}$ gives a linear system for finding $a_{k}$ :

$$
\begin{aligned}
\sum_{k=1}^{K} C_{k} a_{k} & =\sum_{m=1}^{M} A_{m p} w_{m} b_{m}, \\
C_{k} & =\left(\sum_{m=1}^{M} w_{m} A_{m k} A_{m p}\right), \quad p=1, \ldots K
\end{aligned}
$$

A QR decomposition method is employed to solve this system of equations. The coefficients of the resulting linear symmetric matrix A are pre-computed and stored for each element during the preprocessing stage of the calculation, thus increasing the computational efficiency of the method. If the mesh was to be refined these would automatically require the need to recompute and store this matrix. Having solved numerically the linear system, we can form the reconstruction polynomial (24), where by inserting the coordinates of the Gaussian quadrature points of the surface/edges the extrapolated reconstructed solutions are obtained.

\subsubsection{WENO reconstruction}

The main characteristic of the WENO reconstructions is the usage of reconstruction polynomials from several different stencils and their combination in a non-linear manner. In WENO schemes the actual reconstructed value is a convex combination of reconstructed values from stencils, with nonlinear (solution-adaptive) WENO weights [24, 44, 66]. These nonlinear weights are constructed from the linear (constant) weights by taking into account the smoothness of the solution in each of the reconstruction stencils. The resulting methods are uniformly high-order accurate, while maintaining nonoscillatory behaviour in regions with sharp gradients. The WENO reconstruction used in the present study is based on the implementation of [54], where the maximum number of directional stencils is usually equal to the number of faces of each cell. The number of admissible stencils gets smaller in the presence of solid boundaries, as shown in Fig. 4, By solid boundaries for the present study and 
encountered test cases a slip-wall treatment is used where all the variables are set equal to the left-state (considered cell reconstructed extrapolated variables at the faces/edges), apart from the normal velocity component where it's sign is reversed.

The WENO reconstruction polynomial is now defined as a non-linear combination of reconstruction polynomials $p_{m}(\xi, \eta, \zeta)$, obtained by using individual stencils $\mathcal{S}_{m}$

$$
p_{\mathrm{weno}}=\sum_{m=0}^{m_{s}} \omega_{m} p_{m}(\xi, \eta, \zeta) .
$$

Substituting the form of the individual polynomial (24) corresponding to the stencil $\mathcal{S}_{m}$

$$
p_{m}(\xi, \eta, \zeta)=\sum_{k=0}^{K} a_{k}^{(m)} \phi_{k}(\xi, \eta, \zeta),
$$

and using the condition $\sum_{m} \omega_{m} \equiv 1$, we obtain

$$
\begin{aligned}
p_{\text {weno }} & =\sum_{m=0}^{m_{s}} \omega_{m}\left(\sum_{k=0}^{K} a_{k}^{(m)} \phi_{k}(\xi, \eta, \zeta)\right) \\
& =\bar{U}_{0}+\sum_{m=0}^{m_{s}} \omega_{m}\left(\sum_{k=1}^{K} a_{k}^{(m)} \phi_{k}(\xi, \eta, \zeta)\right) .
\end{aligned}
$$

Re-arranging yields

$$
\begin{aligned}
p_{\text {weno }} & =\bar{U}_{0}+\sum_{k=1}^{K}\left(\sum_{m=0}^{m_{s}} \omega_{m} a_{k}^{(m)}\right) \phi_{k}(\xi, \eta, \zeta) \\
& \equiv \bar{U}_{0}+\sum_{k=1}^{K} \tilde{a}_{k} \phi_{k}(\xi, \eta, \zeta),
\end{aligned}
$$

where $\omega_{m}$ are the non-linear weights and $\tilde{a}_{k}$ are the reconstructed degrees of freedoms. The nonlinear weights $\omega_{m}$ following the approaches of [13, 54] are defined as

$$
\omega_{m}=\frac{\gamma_{m}}{\sum_{m=0}^{m_{s}} \gamma_{m}}, \quad \gamma_{m}=\frac{d_{m}}{\left(\varepsilon+I S_{m}\right)^{p}},
$$

where $I S_{m}$ indicates how smooth the solution is on the stencil; $d_{m}$ are the linear weights and $\varepsilon$ is a small number used to avoid division by zero. The central stencil is assigned a large linear weight $d_{0}=10^{2} \ldots 10^{5}$ and the sectorial stencils are assigned smaller weights $d_{m}=1$. The typical values used for $\varepsilon$ is $10^{-6}$ and $p=4$; for details see [12]. 
The smoothness indicators $I S_{m}$ can be computed in a mesh-independent manner as

$$
I S_{m}=\sum_{1 \leq|\beta| \leq r} \int_{V_{0}^{\prime}}\left(D^{\beta} p_{m}(\xi, \eta, \zeta)\right)^{2} d \xi d \eta d \zeta,
$$

where $\beta$ is a multi-index [19, 41], $r$ is the order of the polynomial and $D$ is the derivative operator. The smoothness indicators are quadratic functions of the degrees of freedom $a_{k}^{(m)}$, hence they can be expressed in terms of the universal oscillation indicator matrix [13] . For efficiency, it can be precomputed and stored at the beginning of the calculations for each element $V_{i}$. However it is important to point that the WENO weights are constantly changing (i.e., they are different for every Gaussian quadrature point at every time level).

For the non-hydrostatic equations considered in this study instead of performing the WENO reconstruction for every component of the conserved vector $\mathbf{U}$, the reconstruction is carried out in characteristic variables since they are less prone to spurious oscillations in the proximity of sharp gradients as [13, 54].

\subsubsection{Numerical fluxes and Source term}

Since for each computational cell the point-wise values of the conserved vector $\mathbf{U}$ are replaced by high-order reconstruction polynomials the numerical flux for the face $A_{j}$ of cell $V_{i}$ is discontinuous at each Gaussian point $\beta$. The values $\mathbf{U}_{\beta}^{-}$and $\mathbf{U}_{\beta}^{+}$correspond to the reconstructed value of cell $V_{i}$ and its adjacent neighbour $V_{i^{\prime}}$. In upwind finite-volume methods the discontinuity is replaced at each Gaussian integration point by using a monotone function of left and right boundary extrapolated values so that (15) can be re-written as

$$
\mathbf{K}_{i j} \approx \sum_{\beta} \mathbf{F}_{n, j}\left(\mathbf{U}_{\beta}^{-}, \mathbf{U}_{\beta}^{+}\right) \omega_{\beta}\left|A_{j}\right|
$$

The function $\tilde{\mathbf{F}}_{n, j}\left(\mathbf{U}_{\beta}^{-}, \mathbf{U}_{\beta}^{+}\right)$is the Riemann solver [51]. Employing the rotational invariance property of the Euler equations [51], for each face $A_{j}$ the normal projection of the flux tensor $\mathbf{F}_{n, j}$ is replaced by

$$
\mathbf{F}_{n, j}=\mathbf{T}^{-1} \mathbf{F}\left(\mathbf{T}_{j} \mathbf{U}\right),
$$

where $\mathbf{T}_{j}$ is the rotation matrix for face $j$. Re-writing (37) for $\mathbf{K}_{i j}$ gives 


$$
\begin{aligned}
\mathbf{K}_{i j} & =\sum_{\beta} \mathbf{F}_{n, j}\left(\mathbf{U}_{\beta}^{-}, \mathbf{U}_{\beta}^{+}\right) \omega_{\beta}\left|A_{j}\right| \\
& =\sum_{\beta} \mathbf{T}^{-\mathbf{1}} \mathbf{F}\left(\hat{\mathbf{U}}_{L}, \hat{\mathbf{U}}_{R}\right) \omega_{\beta}\left|A_{j}\right|,
\end{aligned}
$$

where $\hat{\mathbf{U}}_{j}$ is the rotated conserved variable and

$$
\hat{\mathbf{U}}_{L}=\mathbf{T}_{j} \mathbf{U}_{\beta}^{-}, \quad \hat{\mathbf{U}}_{R}=\mathbf{T}_{j} \mathbf{U}_{\beta}^{+} .
$$

The flux function for the Gaussian point $\beta$ results in the one-dimensional Riemann initial value problem

$$
\begin{aligned}
\frac{\partial}{\partial t} \hat{\mathbf{U}}+\frac{\partial}{\partial s} \hat{\mathbf{F}}=\mathbf{0}, \quad \hat{\mathbf{F}} & =\mathbf{F}(\hat{\mathbf{U}}), \\
\hat{\mathbf{U}}(s, 0) & = \begin{cases}\hat{\mathbf{U}}_{L}, & s<0, \\
\hat{\mathbf{U}}_{R}, & s>0\end{cases}
\end{aligned}
$$

In the present study the Harten-Lax-Van Leer-Contact (HLLC) Riemann solver of Toro [51] is employed. The numerical source term $\mathbf{S}_{\mathbf{i}}$ is approximated by using a Gaussian quadrature rule of the same order of accuracy as the order of the polynomial of the reconstruction

$$
\mathbf{S}_{\mathbf{i}} \approx \frac{1}{\left|V_{i}\right|} \sum_{\beta} \mathbf{S}\left(x_{\beta}, y_{\beta}, z_{\beta}, t\right) w_{\beta},
$$

where $x_{\beta}, y_{\beta}, z_{\beta}$ is the volumetric Gaussian quadrature points and $w_{\beta}$ is the Gaussian weights of $r$-order of accuracy.

\subsection{Temporal discretisation}

Having constructed the numerical fluxes $\mathbf{F}_{n, j}$ as expressed in the semi-discrete conservative formulation, the next step involves the advancement of the solution in time. The explicit Strong Stability Preserving (SSP) Runge-Kutta $3^{r d}$-order method [20] has been employed for the time integration

$$
\begin{gathered}
\mathbf{U}_{\mathbf{i}}^{1}=\mathbf{U}_{\mathbf{i}}^{n}+\Delta t \cdot \mathbf{R}_{i}\left(\mathbf{U}_{\mathbf{i}}^{n}\right) \\
\mathbf{U}_{\mathbf{i}}{ }^{2}=\frac{3}{4} \mathbf{U}_{\mathbf{i}}^{n}+\frac{1}{4} \mathbf{U}_{\mathbf{i}}^{1}+\frac{\Delta t}{4} \cdot \mathbf{R}_{i}\left(\mathbf{U}^{1}\right) \\
\mathbf{U}_{\mathbf{i}}^{n+1}=\frac{1}{3} \mathbf{U}_{\mathbf{i}}^{n}+\frac{2}{3} \mathbf{U}_{\mathbf{i}}{ }^{2}+\frac{2 \Delta t}{3} \cdot \mathbf{R}_{i}\left(\mathbf{U}_{\mathbf{i}}{ }^{2}\right)
\end{gathered}
$$


with $\mathbf{R}_{i}$ given by (13).

The time step $\Delta t$ is computed as follows

$$
\Delta t=K \min _{i} \frac{h_{i}}{S_{i} \cdot V_{i}}
$$

where $h_{i}$ is the radius of the inscribed sphere of each cell $i$ and $V_{i}$ its corresponding volume, $K \leq 1 / 3$ is the CFL number for unsplit finite-volume schemes [51] , and $S_{i}$ is the maximum propagation speed in each cell $i$ given by

$$
S_{i}=s p_{x} \cdot n_{x}+s p_{y} \cdot n_{y}+s p_{z} \cdot n_{z}
$$

where

$$
s p_{x}=|u+a|, \quad, s p_{y}=|v+a|, \quad s p_{x}=|w+a|,
$$

with $\mathbf{n}=\left(n_{x}, n_{y}, n_{z}\right)$ being the outward unit normal vector and $a$ is the speed of sound.

\section{RESULTS}

This section presents the results obtained with the WENO schemes for a series of 2D and 3D test cases of a stratified atmosphere.

\subsection{D Robert Smooth Bubble}

After its introduction [42], the Robert smooth bubble problem is considered as a standard benchmark in NWP. The evolution of a warm bubble in a constant potential temperature environment is investigated, and since the bubble is warmer than the ambient air, it rises and due to shearing motion it forms a mushroom shaped structure as shown in Fig. 6. The two-dimensional, non-hydrostatic compressible Euler equations (1D) are numerically solved in the computational domain $1000 \mathrm{~m} \times 1500 \mathrm{~m}$ with $t \in[0,800] s$ with no flux boundary conditions. The initial condition [42] corresponds to a warm bubble centered at $(500,260) m$, which represents a perturbation of the potential temperature when the rest of the domain is in hydrostatic balance, in order to trigger the movement of the warm bubble as follows: 


$$
\theta^{\prime}=\left\{\begin{array}{cl}
0 & \text { for } \quad r<r_{c} \\
0.25\left(1+\cos \left(\frac{\pi r}{r_{c}}\right)\right) & \text { for } \quad r \geq r_{c}
\end{array},\right.
$$

where $r$ is the distance from the centre of the bubble

$$
r=\sqrt{\left(x-x_{c}\right)^{2}+\left(z-z_{c}\right)^{2}}
$$

where $\pi$ is the trigonometric constant; $r_{c}=250 \mathrm{~m}$ is the radius of the bubble; and the potential temperature $\bar{\vartheta}$ is constant at $300 \mathrm{~K}$. The unstructured meshes used consist of arbitrary triangular elements as shown in Fig. 5 and have resolutions of a $25 \mathrm{~m}, 6 \mathrm{~m}$ and $3 \mathrm{~m}$. The WENO3 and WENO5 schemes using characteristics based reconstructions are used, with a CFL number of 0.9.

From the obtained computational results as shown in Fig. 6 and Fig. 7 it can be noticed that longwave oscillations of the interface occur forming smaller vortices, the spatial symmetry of the solution is completely lost and that higher-order WENO schemes resolve more of these small scale vortices.

Regarding the long-wave oscillations of the interface it can be attributed to the the shear flow interaction between the interface and the surrounding unperturbed air, which in turn give rise to the Kelvin-Helmholtz instabilities that lead to turbulent structures. There have been numerous extensive numerical experiments [21, 35, 42, 64] to investigate this problem and to date the exact solution remains unknown. The spatial symmetry of the solution is lost due to the fact that the mesh is not symmetric, the initial profile is approximated by a Gaussian quadrature rule of the same order as the polynomial order used for the reconstruction, and finally due to the multidimensional reconstruction of the present algorithms. This behaviour was noticed in the application of WENO finite volume schemes for the double vortex pairing problem, but the presence of viscosity resulted in less pronounced asymmetries [53]. The fact that each time the mesh is refined,or a higher-order scheme (due to the Gaussian quadrature) is used the initial perturbations change slightly. In return these perturbations according to [21] grow exponentially in time, hence the perturbations have larger spatial scales that the initial perturbations.

As the grid resolution is refined or as the spatial order of the WENO schemes is increased the numerical dissipation is reduced hence more structures are resolved. What is of essence though is if this structures are just artificial or if they are part of the correct solution. Along these lines it must be stressed that the subject WENO schemes have demonstrated their robustness for treating strongly discontinuous flow problems on arbitrary unstructured meshes [52], therefore it can not be attributed to the WENO schemes discontinuous capturing capabilities. On the contrary WENO schemes for this 


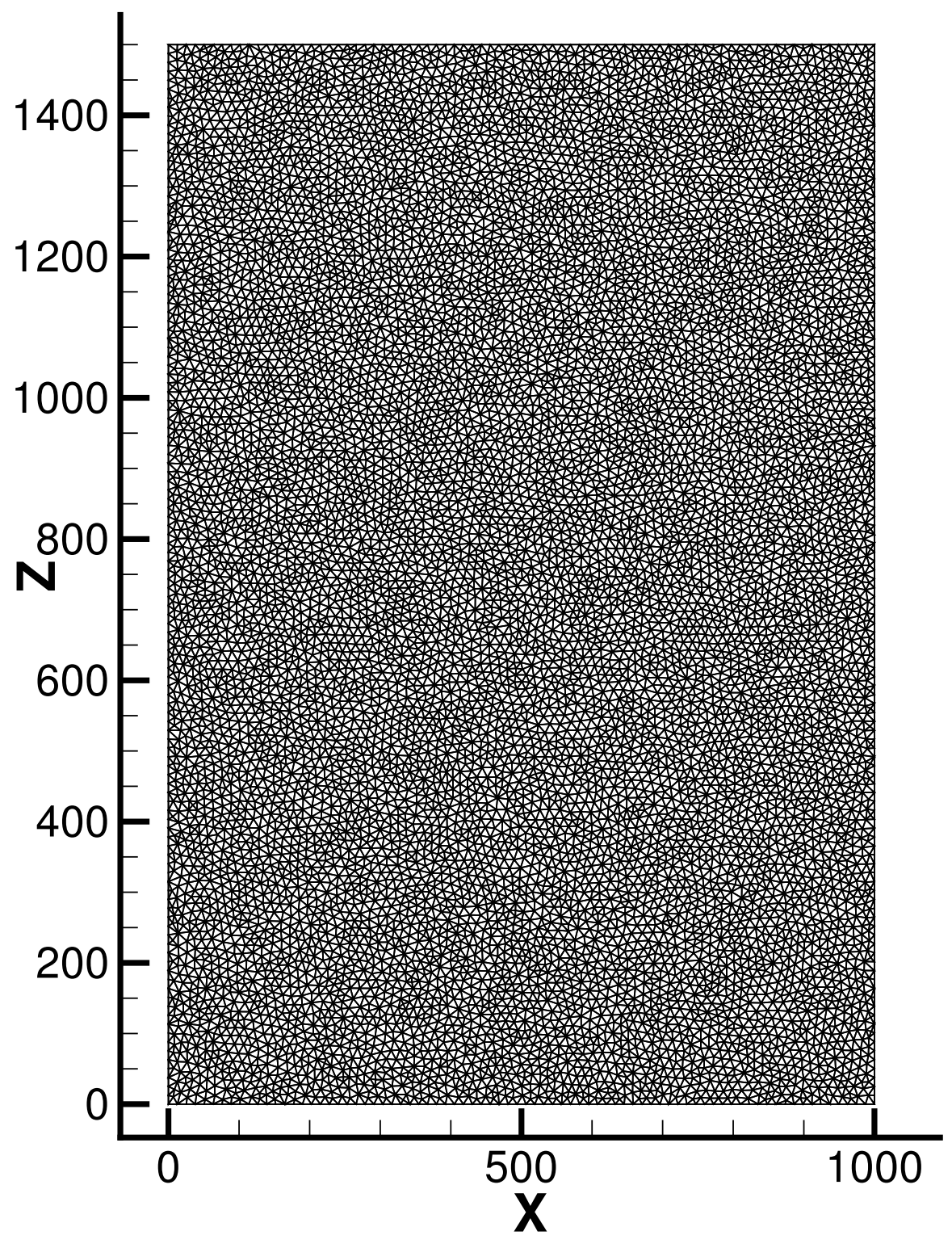

FIG. 5: Unstructured mesh used for the rising thermal bubble case.

discontinuous problem remain stable as witnessed by [40]. However for other approaches that do not possess discontinuity capturing capabilities a form of artificial viscosity would be required firstly to stabilise the solution and secondly for obtaining a converged solution [35]. At the same time it can be realised that some of these perturbations are actually the correct solution [35, 64] hence controlling the 


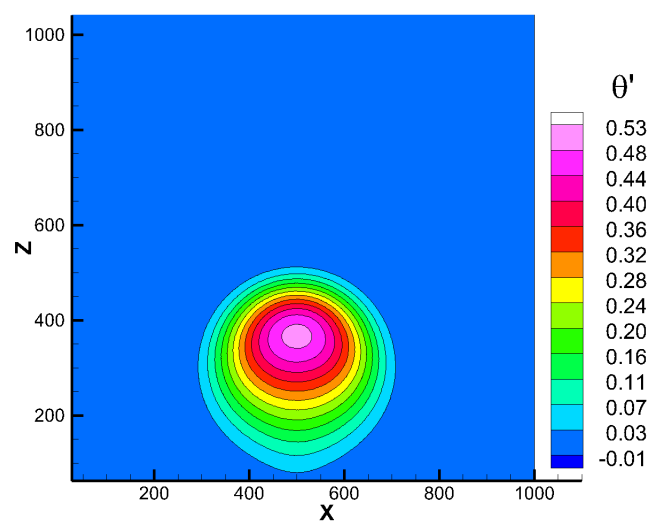

(a) $t=172 \mathrm{~s}$

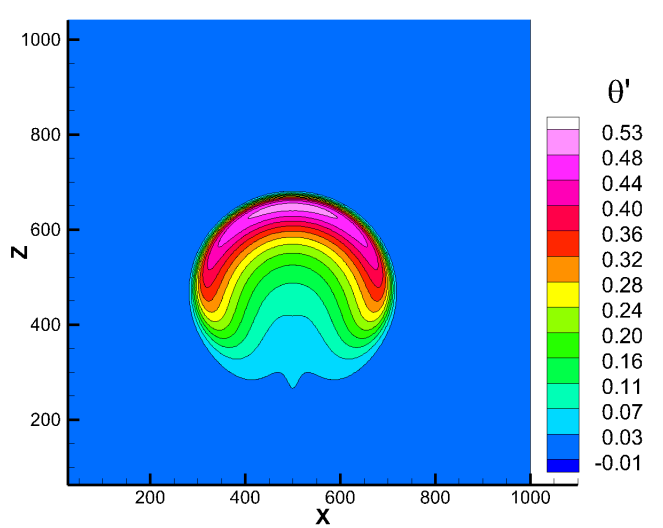

(c) $t=400 \mathrm{~s}$

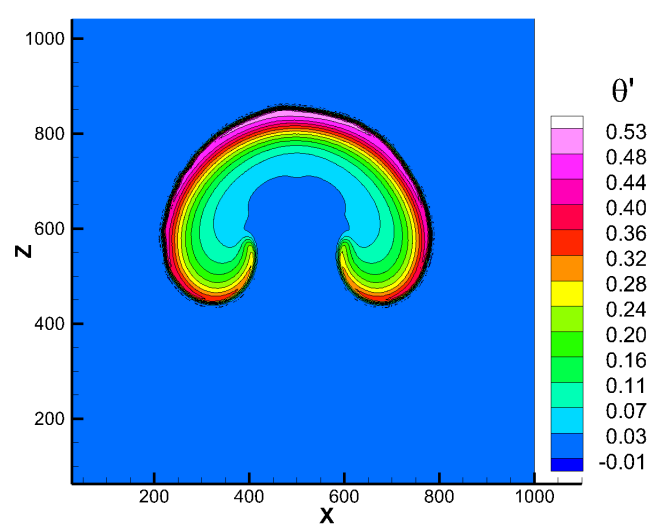

(e) $t=600 \mathrm{~s}$

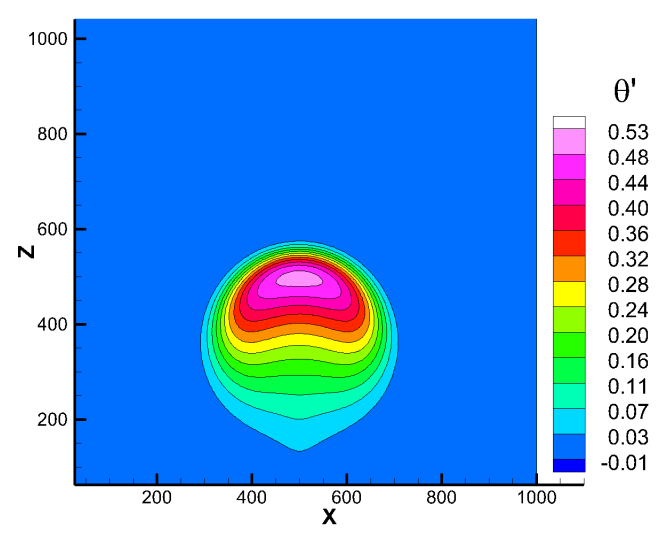

(b) $t=271 \mathrm{~s}$

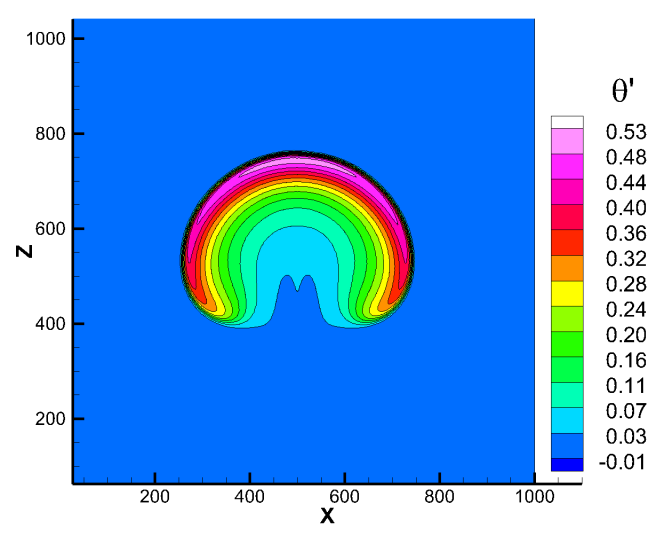

(d) $t=480 \mathrm{~s}$

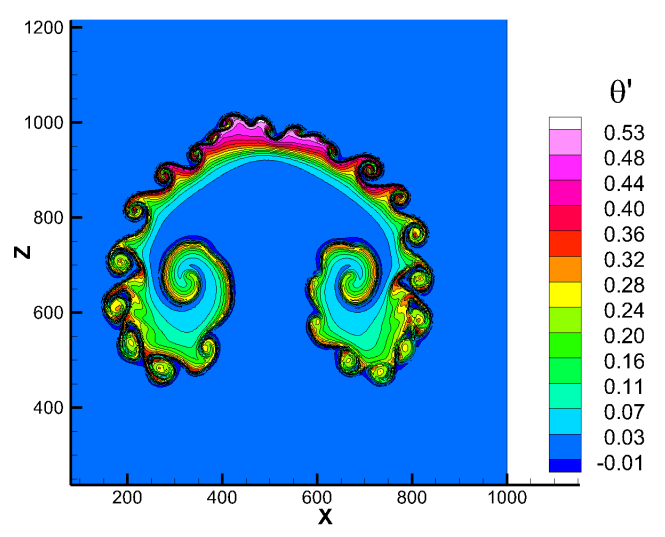

(f) $t=800 \mathrm{~s}$

FIG. 6: Potential temperature perturbation $\theta^{\prime}(K)$ at various instants for the 2D Robert smooth bubble test on an unstructured mesh of $6 m$ resolution using a WENO5 scheme. 
amount of artificial viscosity can be quite challenging. But atmospheric models should be used with little to no artificial viscosity since most of the time they are used completely under-resolved.

\subsection{Straka Density Current}

The Straka [49] density current test problem is also considered to be a well established test for NWP. This problem concerns the evolution of a cold bubble in a neutrally stratified atmosphere, where the bubble descends to the ground due to negative buoyancy and when it hits the ground it is rolled up and a front is created, as shown in Fig. 9. The two-dimensional, non-hydrostatic compressible Euler equations (1) are numerically solved in the computational domain $25600 \mathrm{~m} \times 6400 \mathrm{~m}$ and $t \in$ $[0,900] s$ with no flux boundary conditions. The initial condition [42] corresponds to a cold bubble of size $\left(x_{r}, z_{r}\right)=(4000,2000) m$ centred at $(0,3000) m$, which represents a perturbation of the potential temperature to trigger the movement of the cold bubble as follows:

$$
\begin{gathered}
\theta^{\prime}=\left\{\begin{array}{cc}
0 & \text { for } r<r_{c} \\
-7.5\left(1+\cos \left(\frac{\pi r}{2}\right)\right) & \text { for } r \geq r_{c}
\end{array},\right. \\
r=\sqrt{\left(\frac{x-x_{c}}{r_{c}}\right)^{2}+\left(\frac{z-z_{c}}{r_{c}}\right)^{2}} .
\end{gathered}
$$

The subject test problem is modified from its original form and has been simulated without the explicit viscosity of $75 \frac{m^{2}}{s}$ similarly to [2, 31, 40]. Since the problem is inviscid, nonlinear and unsteady, without explicit viscosity the grid-convergence of the solution is not expected, but at the same time the stabilising properties of upwind schemes is of importance, as it has been the motivation for numerous studies [2, 31, 40]. The unstructured meshes used consist of both quadrilaterals near the ground and arbitrary triangular elements as shown in Fig. 8 and have resolutions of a $100 \mathrm{~m}, 50 \mathrm{~m}$ and $25 \mathrm{~m}$. The WENO3 and WENO5 schemes based on characteristics variables reconstructions are employed, with a CFL number of 0.9 .

From the obtained computational results as shown in Fig. 9, Fig. 10 and Fig. 11 it can be noticed that the number of resolved Kelvin-Helmholtz rotors differs between different resolutions, schemes and that the location of the front for all simulations is close to $15 \mathrm{~km}$. As in the 2D Robert smooth bubble test case the initial profile changes when the mesh is refined or when the numerical scheme is changed due to the inherent high-order Gaussian quadrature approximation of the volume, surface and line integrals 


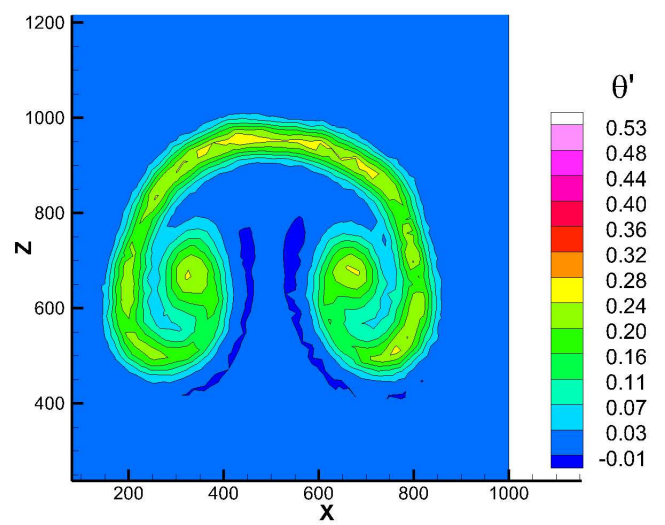

(a) $25 \mathrm{~m}$ WENO3

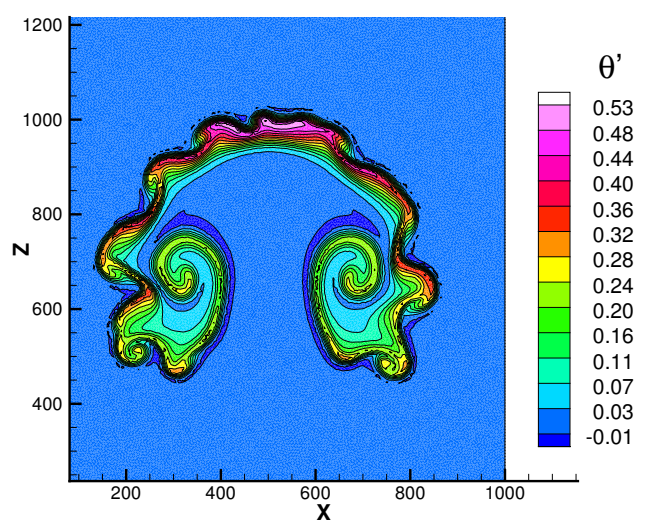

(c) $6 \mathrm{~m}$ WENO3

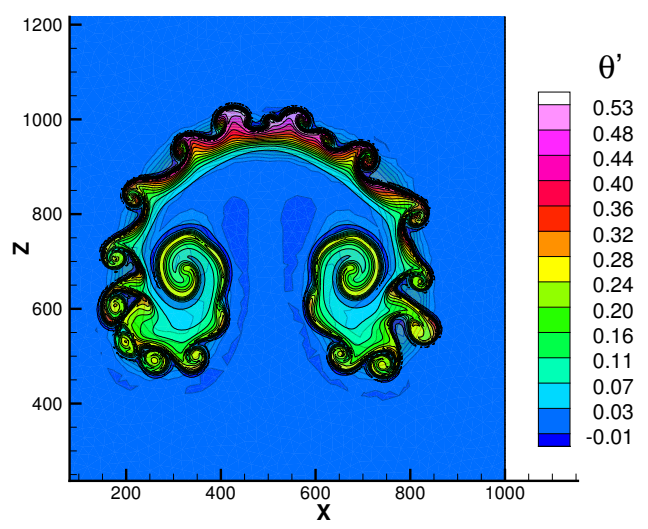

(e) $3 \mathrm{~m}$ WENO3

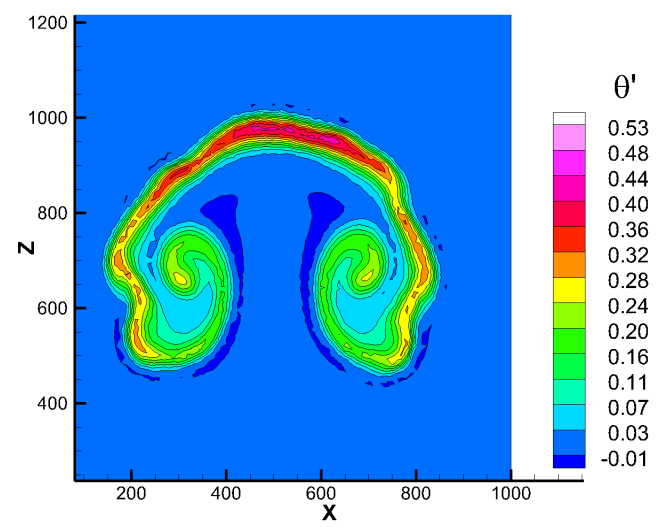

(b) $25 \mathrm{~m}$ WENO5

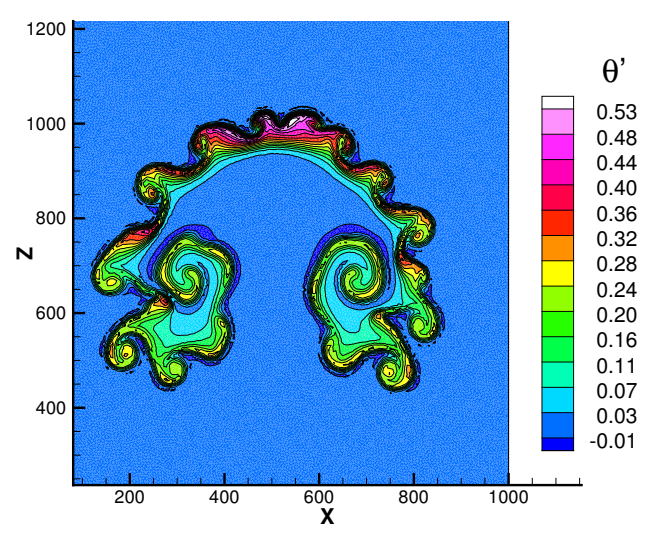

(d) $6 \mathrm{~m}$ WENO5

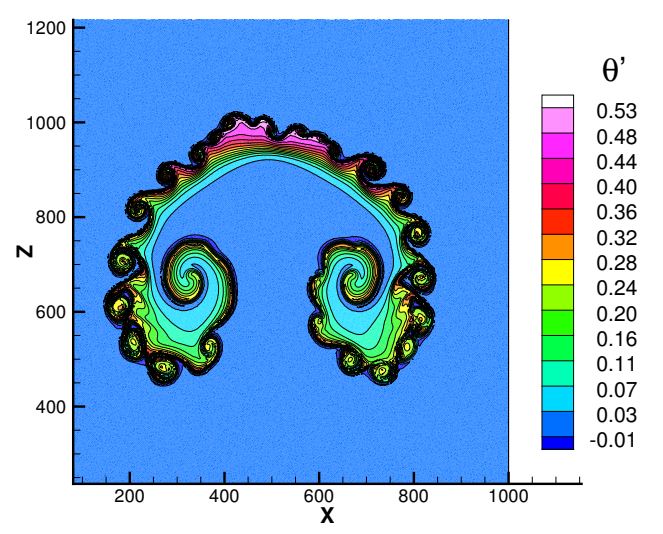

(f) $3 \mathrm{~m}$ WENO5

FIG. 7: Potential temperature perturbation $\theta^{\prime}(K)$ at $t=800 \mathrm{sec} 2 \mathrm{D}$ Robert smooth bubble test at different grid resolutions with WENO3 and WENO5 schemes. 


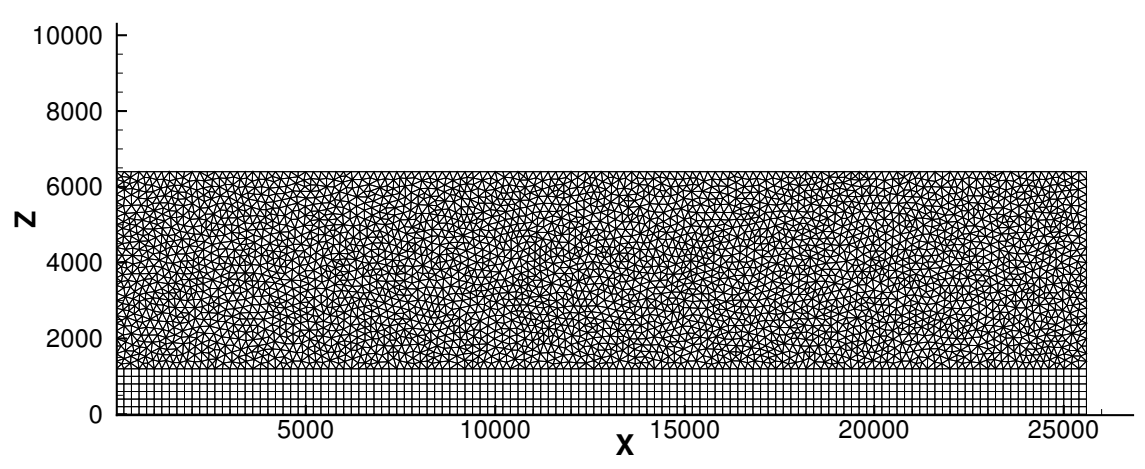

FIG. 8: Unstructured hybrid mesh used for the density current test case.

associated with WENO Finite Volume schemes [52]; therefore without explicit viscosity it can not be expected to reach to a grid-converged solution, but at the same time WENO schemes without explicit viscosity will not eventually blow up as other approaches [16]. The results suggest that it is not due to the discontinuity-capturing capabilities of the WENO schemes not working properly, but it is rather because of the low dissipation of the present schemes that the number of the resolved features increase since the numerical dissipation is greatly reduced. On the other hand the resolutions employed at this study might be extreme $(25 \mathrm{~m})$ to justify the use of high-order schemes when the original viscosity of the test problem which is $75 \mathrm{~m}^{2} / \mathrm{s}$ can be considered relatively large compared to the grid resolution. What is important though is that the present numerical schemes remain stable at this CFL number, which is not the case for other central-schemes without explicit viscosity as documented in [2].

The present shear triggered structures show great similarities to these witnessed in another recent study [32], where the highest resolution to date to the best of our knowledge of $12.5 \mathrm{~m}$ has been used. Comparing quantitatively the performance of the subject WENO schemes with other schemes in the literature in terms of $\theta^{\prime}$ extrema, and front position at $t=900 \mathrm{~s}$ we notice firstly that the variational multiscale stabilized finite element method (VMS) scheme [31], the f-wave[2] and the WENO schemes are the only ones used without explicit diffusion. The WENO schemes have less oscillations in terms of $\theta_{\max }^{\prime}$ than the VMS (FE) that do not employ a discontinuity capturing method, but at the same time the WENO schemes seem to be bounded in terms of the $\theta_{\min }^{\prime}$, which is expected due to the initial profile corresponding to colder air by $15^{\circ} \mathrm{C}$. In contrast the numerical frameworks that utilise explicit viscosity, their $\theta_{\max }^{\prime}$ are bounded at 0 .

What is striking though is that the total variation in terms of front location between the $25 \mathrm{~m}$ resolu- 


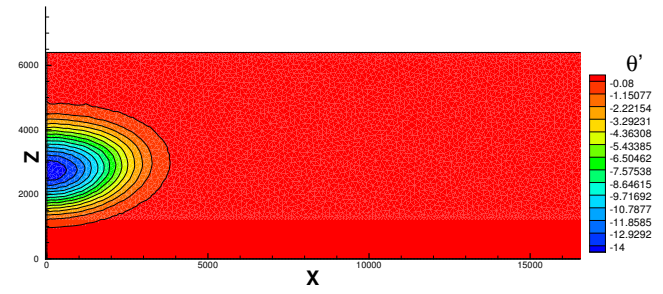

(a) $t=10 \mathrm{~s}$

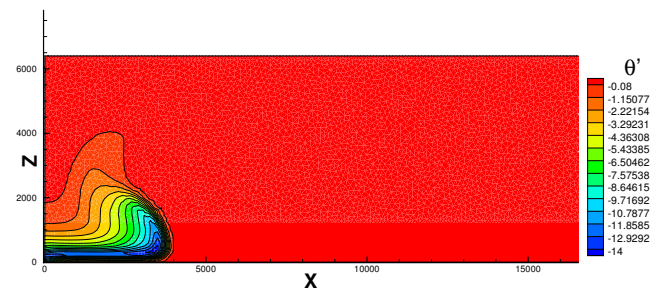

(c) $t=300 \mathrm{~s}$

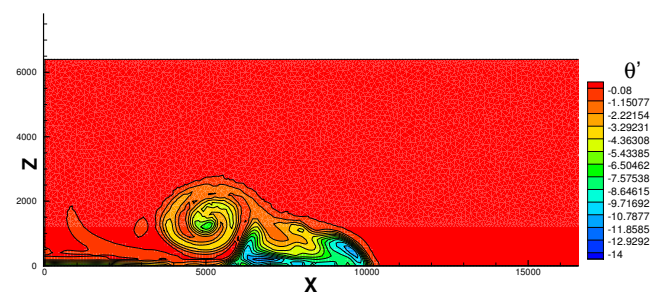

(e) $t=600 \mathrm{~s}$

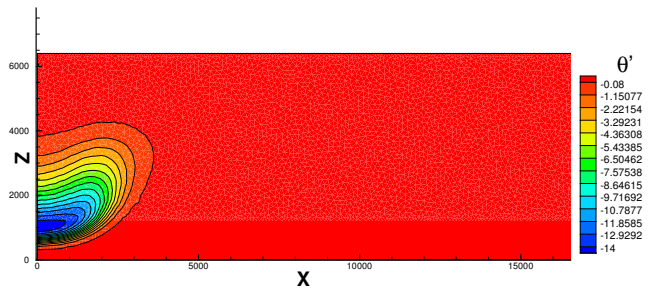

(b) $t=180 \mathrm{~s}$

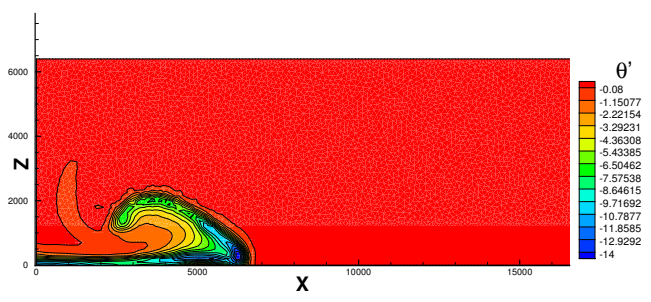

(d) $t=420 \mathrm{~s}$

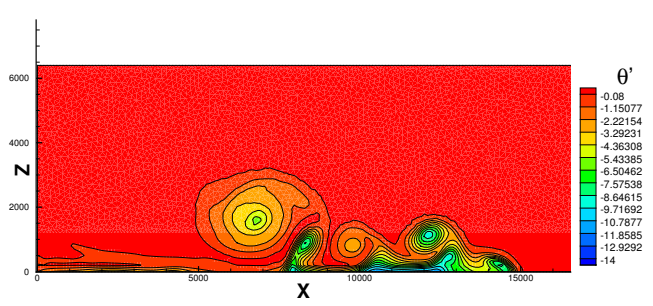

(f) $t=900 \mathrm{~s}$

FIG. 9: Potential temperature perturbation $\theta^{\prime}(K)$ at various instants for the density current test on a hybrid unstructured mesh of $100 \mathrm{~m}$ resolution with WENO5 scheme.

tion simulations the WENO schemes have a spread of $170 \mathrm{~m}$ and $250 \mathrm{~m}$ across 3 different resolutions, where three different approaches used by Straka [49] at the same resolution with explicit viscosity have a spread of $590 \mathrm{~m}$ and [16] approach being the only one that is converged across two different numerical schemes (SE) and (DG). This leads us to conclude that for high-order schemes which their numerical viscosity is considerably smaller than the explicit viscosity for the subject test problem one should get converged solution with minimum variation across different resolutions as in [16]. On the other hand if the numerical schemes need additional forms of artificial viscosity for stabilisation, obtaining the correct converged solution can be quite challenging, since the best artificial diffusion to be used in atmospheric simulations remains a very open topic of study. The behaviour of the present WENO schemes in terms of numerical dissipation, has been closely linked to the type of elements em- 


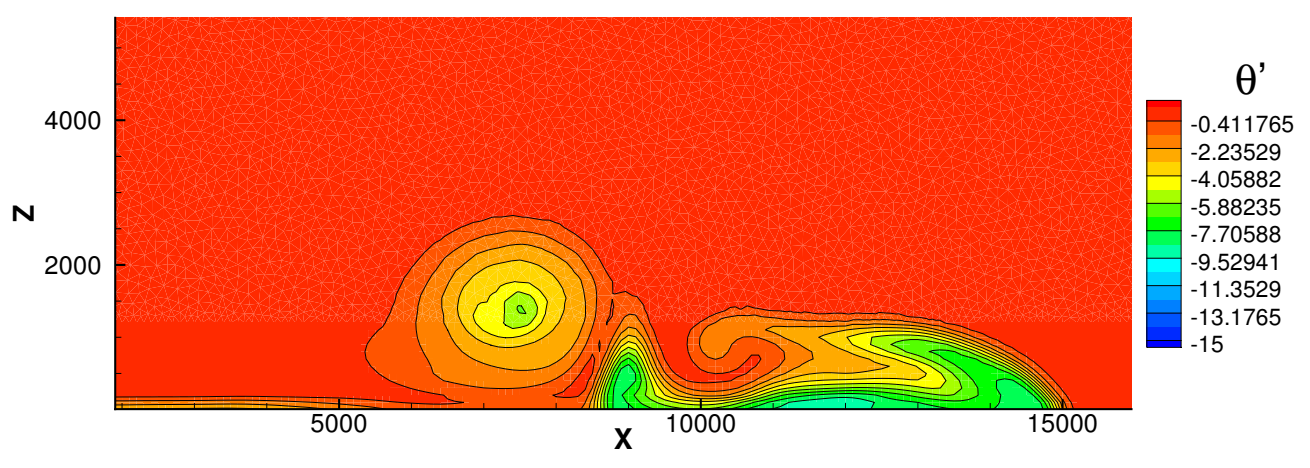

(a) 100m WENO3

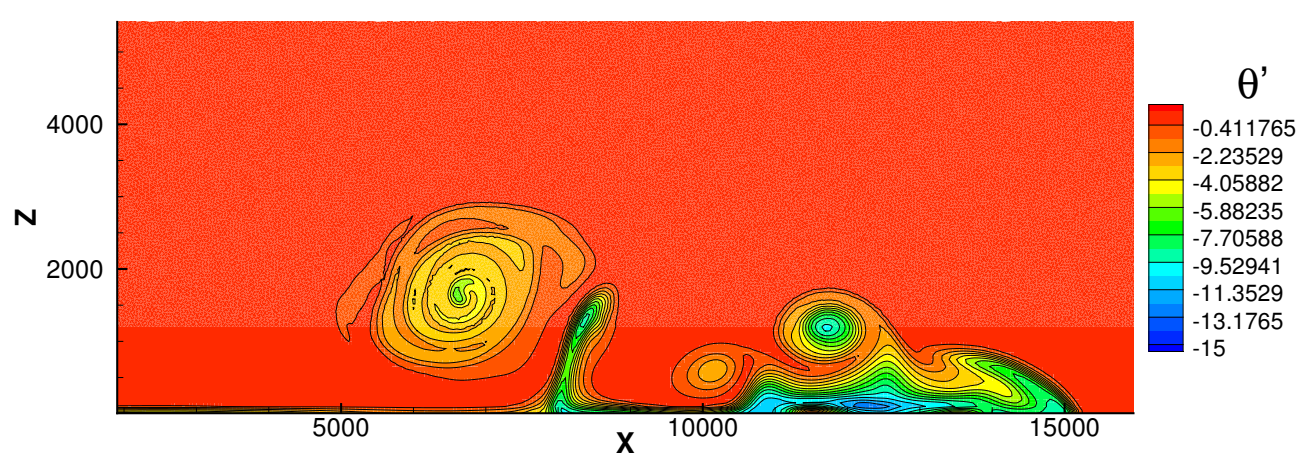

(b) $50 \mathrm{~m} \mathrm{WENO} 3$

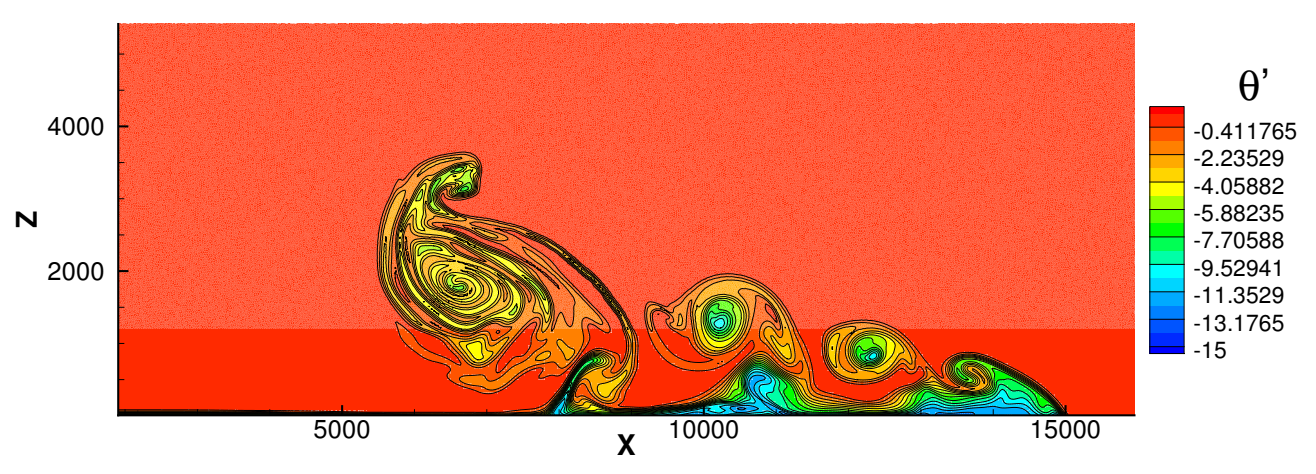

(c) $25 \mathrm{~m} \mathrm{WENO} 3$

FIG. 10: Potential temperature perturbation $\theta^{\prime}(K)$ at $t=900 \mathrm{~s}$ for the density current test on a hybrid unstructured meshes of different resolution with WENO3 scheme. 


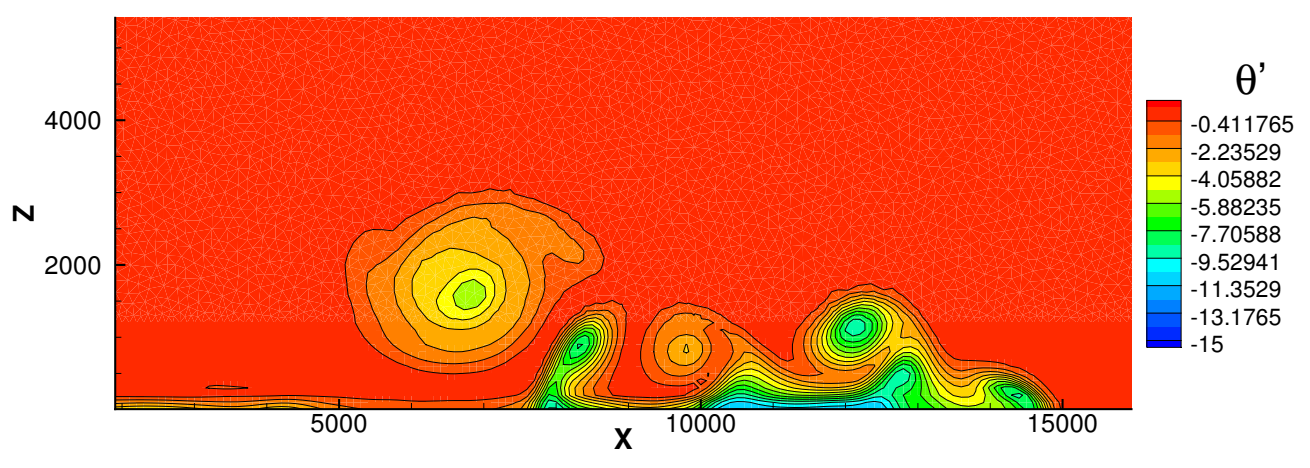

(a) 100m WENO5

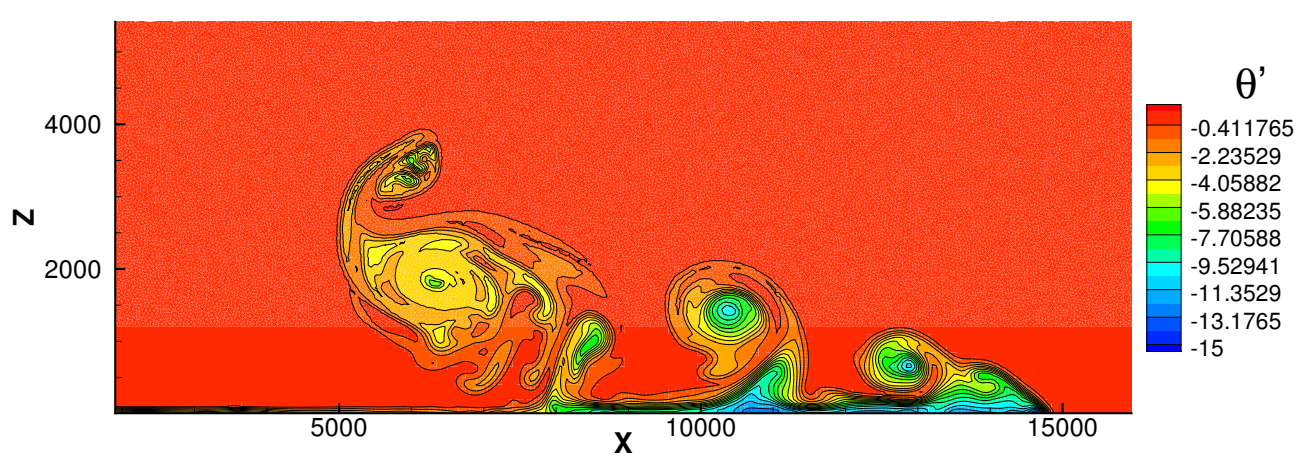

(b) $50 \mathrm{~m}$ WENO5

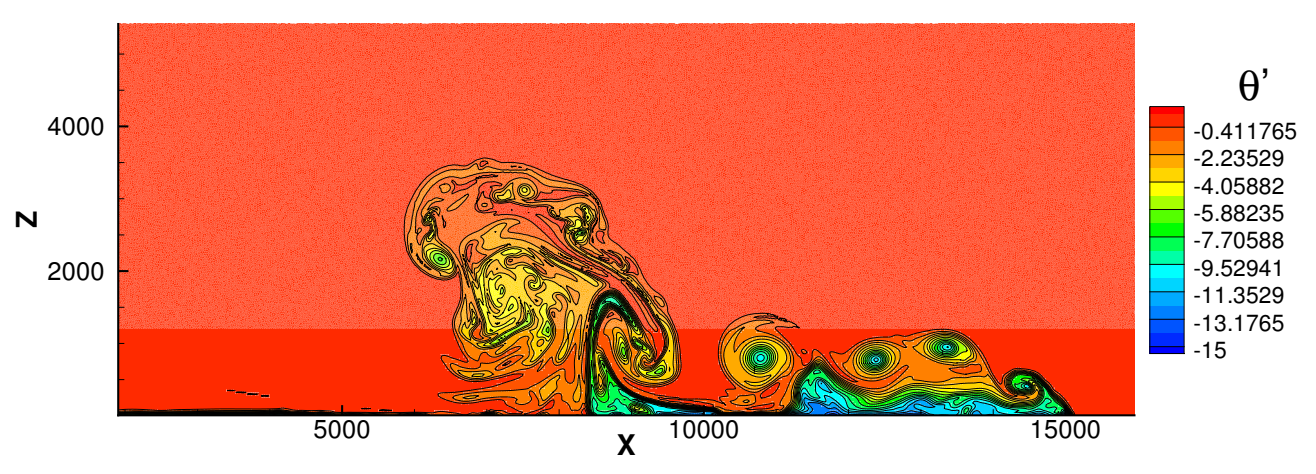

(c) $25 \mathrm{~m}$ WENO5

FIG. 11: Potential temperature perturbation $\theta^{\prime}(K)$ at $t=900 \mathrm{~s}$ for the density current test on a hybrid unstructured meshes of different resolution with a WENO5 scheme. 


\begin{tabular}{c|c|c|c}
\hline Method & $\theta_{\text {min }}^{\prime}$ & $\theta_{\text {max }}^{\prime}$ & Front location (m) \\
\hline WENO3 100m & -9.535 & 0.308 & 14870 \\
WENO5 100m & -11.523 & 0.476 & 14910 \\
WENO3 50m & -13.022 & 0.506 & 14900 \\
WENO5 50m & -14.754 & 0.613 & 15030 \\
WENO3 25m & -13.427 & 0.537 & 14950 \\
WENO5 25m & -14.868 & 0.634 & 15120 \\
f-wave TVD 50m [2] & -9.82 & 0.0089 & 14975 \\
VMS (FE) 25m [31] & -13.98 & 7.81 & 14890 \\
(SEM-4th) 12.5m [32] & - & - & 15056 \\
(SEM-4th) 25m [32] & - & - & 14992 \\
(SEM-4th) 50m [32] & - & - & 14535 \\
(SEM-4th) 100m [32] & - & - & 14325 \\
SE 25m [16] & -8.90 & $1.2 \mathrm{e}-4$ & 14767 \\
DG 25m [16] & -8.90 & $1.2 \mathrm{e}-4$ & 14767 \\
REFC 25m [49] & -9.77 & 0.0 & 14437 \\
REFQ 25m [49] & -10.00 & 0.0 & 14409 \\
PPM 25m [49] & -8.31 & 0.022 & 15027 \\
\hline
\end{tabular}

TABLE I: Comparison of results obtained with the present schemes at various grid resolutions in terms of $\theta^{\prime}$ extrema, and front position at $t=900 \mathrm{~s}$ with other schemes available in literature in the framework of FE, SE and DG for the density current test case.

ployed in the grid as reported in previous studies [3, 4, 9, 52]. Since triangular and tetrahedral elements have demonstrated sufficiently smaller dissipation errors as compared to quadrilateral and hexahedral meshes. Therefore any artificial viscosity/diffusion stabilisation scheme should take into account apart from the grid resolution the grid type employed, due to their inherently different dissipation properties.

balance between numerical dissipation and artificial viscosity/diffusion for stabilization in the context of the developed schemes would be useful, in particular from the point of view of practical recommendations 


\subsection{D Robert Smooth Bubble}

The three dimensional, non-hydrostatic compressible Euler equations (1) are numerically solved in the computational domain $1000 m \times 1000 m \times 1500 m$ and $t \in[0,450] s$ in conjunction with NFBC. The initial condition [42] corresponds to a warm bubble centered at $(500,500,260) \mathrm{m}$, which represents a perturbation of the potential temperature to trigger the movement of the warm bubble as follows:

$$
\theta^{\prime}=\left\{\begin{array}{cl}
0 & \text { for } \quad r<r_{c} \\
1.25\left(1+\cos \left(\frac{\pi r}{r_{c}}\right)\right) & \text { for } \quad r \geq r_{c}
\end{array}\right.
$$

where $r$ is the distance from the centre of the bubble

$$
r=\sqrt{\left(x-x_{c}\right)^{2}+\left(y-y_{c}\right)^{2}+\left(z-z_{c}\right)^{2}},
$$

$\pi$ is the trigonometric constant; $r_{c}=250$ is the radius of the bubble; and the potential temperature $\bar{\vartheta}$ is constant at $300 \mathrm{~K}$. Three unstructured meshes comprising of 19, 175, 64, 000 , and 210, 212 elements have been employed using tetrahedral elements Fig. 12

The main objective of extending this test problem in three dimensions is firstly to assess the performance of the WENO schemes in terms of robustness and accuracy when running this problem at extremely low spatial resolution $30 \sim 100 \mathrm{~m}$. This is more realistic in terms of under resolved gridarrangements used in NWP. Secondly the structure of the solution of the same problem is of importance. The WENO3 and WENO5 schemes using characteristics based reconstructions are used, with a CFL number of 0.9 .

From the obtained computational results as shown in Fig. 13 it can be noticed that the solution does not exhibit the same instabilities of the 2D test case, although the spatial symmetry of the solution is also lost, and that the higher-order WENO schemes provide sharper profile solutions.

Regarding the solution not exhibiting the smaller Kelvin-Helmholtz instabilities associated with the 2D case, this was expected since the dissipation of the numerical schemes in three dimensions and in the much coarser grid resolution is much greater than in $2 \mathrm{D}$. At the same time the under-resolved grid arrangements pose severe challenges for the present WENO schemes. Firstly the multidimensional character of the schemes when combined with arbitrary unstructured meshes for under resolved simulations, the non-oscillatory properties of the WENO schemes are stressed because due to the coarse grid the gradients of the flow variable can become quite sharp, but for the present case they do not seem 


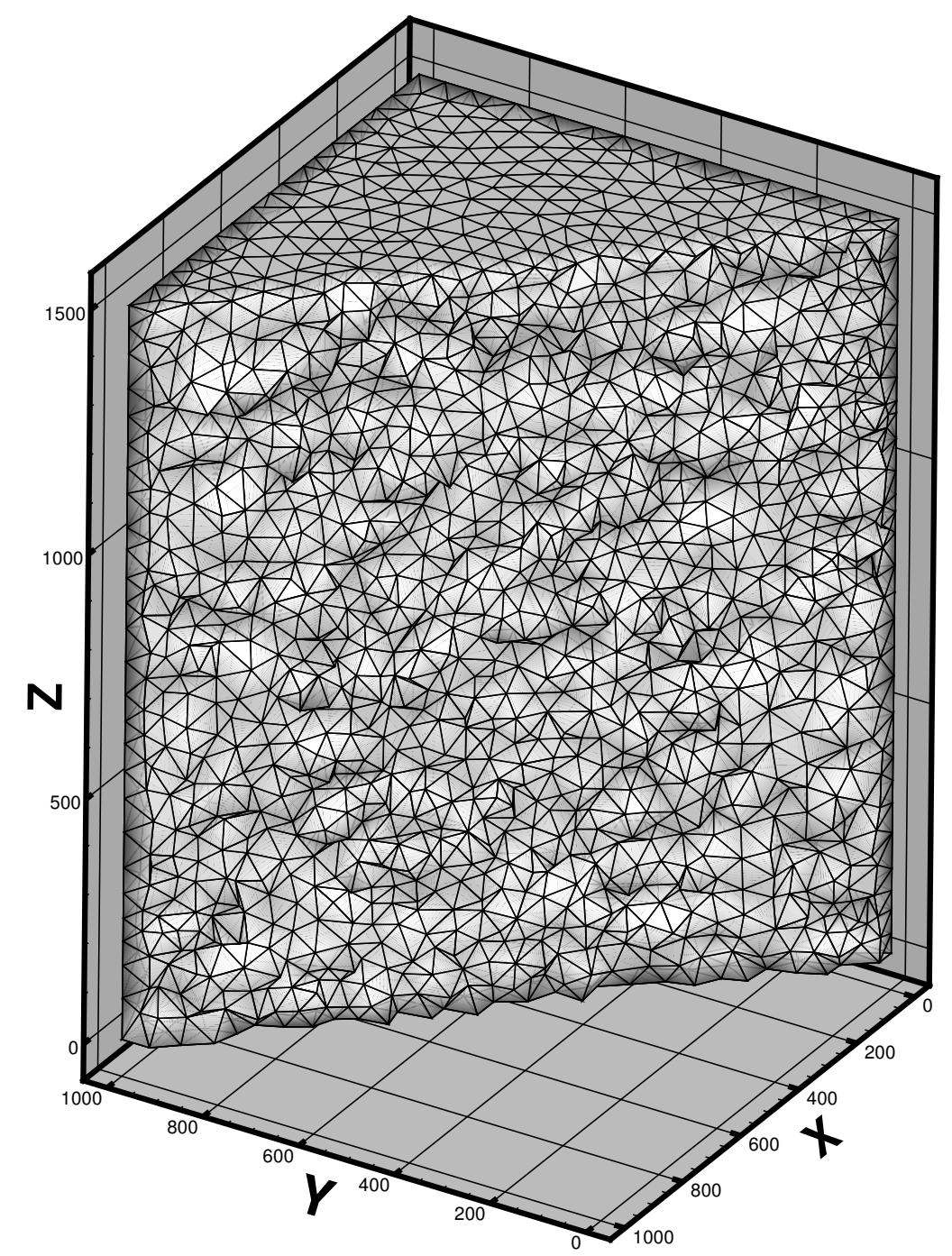

FIG. 12: Cutaway of Unstructured mesh used for the 3D Robert smooth bubble test.

to face any problem in terms or robustness. On the other hand the coarse resolution results in loss of symmetry of the solution, due to the multidimensional character of the reconstruction in a very coarse arbitrary non symmetric grid arrangement, but this loss of symmetry is actually a realistic feature of a series of well established instabilities that get initiated by a perturbed initial profile. The higherorder WENO schemes provide much sharper and accurate results, and when looking at the potential temperature perturbation $\theta^{\prime}(K)$ and vertical velocity $\mathbf{W}^{\prime}(\mathrm{m} / \mathrm{s})$ profiles of 15 , although the solution is not expected to converge without any explicit viscosity due to the inviscid nature of the problem, as the 


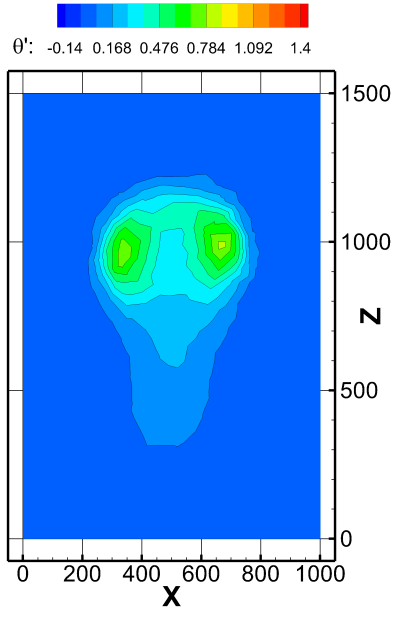

(a) $90 \mathrm{~m}$ WENO3

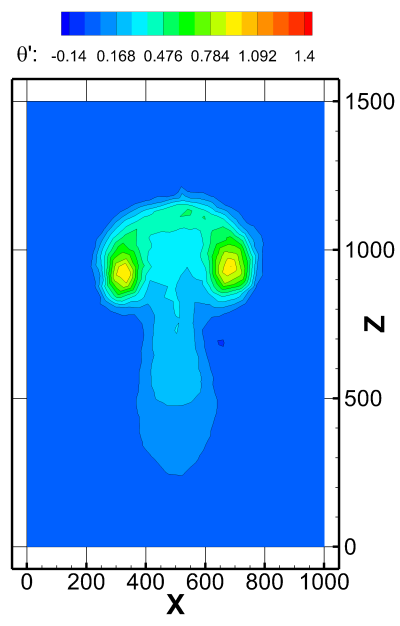

(d) $90 \mathrm{~m}$ WENO5

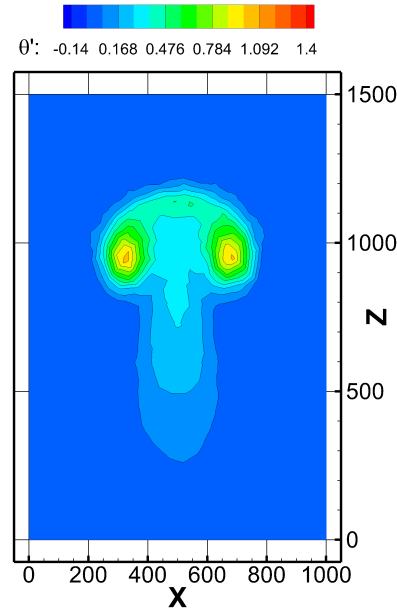

(b) $60 m$ WENO3

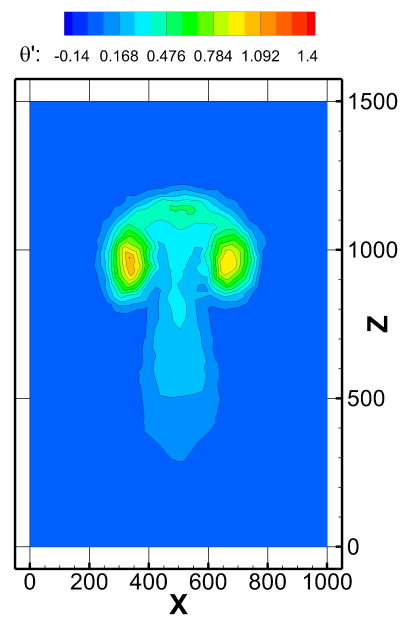

(e) $60 \mathrm{~m}$ WENO5

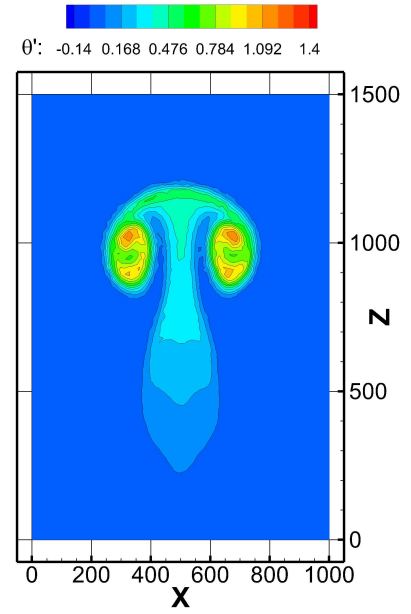

(c) $30 m$ WENO3

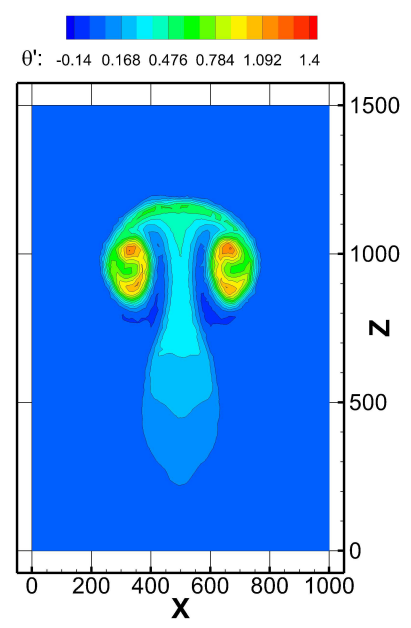

(f) $30 \mathrm{~m}$ WENO5

FIG. 13: Potential temperature perturbation $\theta^{\prime}(K)$ at $t=300 \mathrm{sec}$ and $y=500 \mathrm{~m}$ for the 3D Robert smooth bubble test.

mesh is refined a closer agreement is observed for the vertical velocity and for the potential temperature perturbation. Once again the asymmetry of solution is more pronounced at the coarse grid resolutions.

\subsection{Parallel Performance}

The requirements for atmospheric modelling in a regional or global scale have significantly increased in the last decade. Parallelisation of existing computational methods and software is an active 


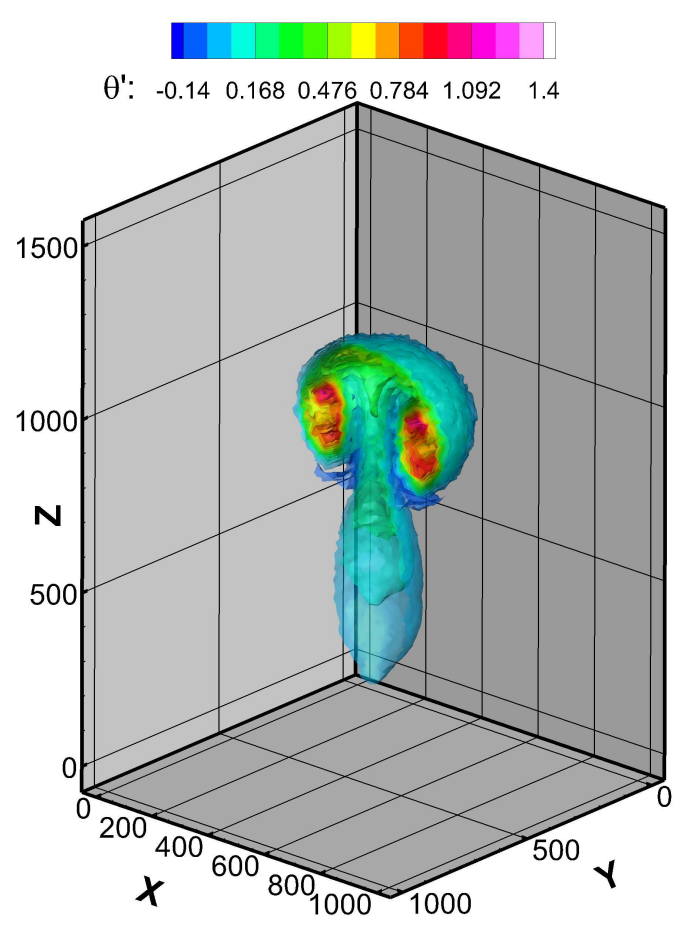

(a) $\theta^{\prime}(K)$

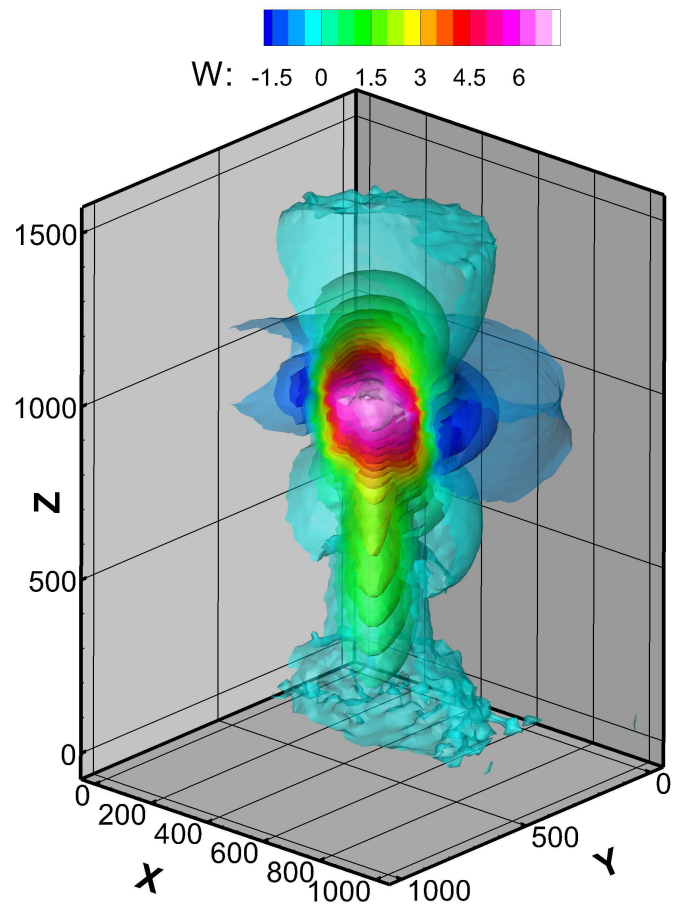

(b) $\mathbf{W}(m / s)$

FIG. 14: Potential temperature perturbation $\theta^{\prime}(K)$ and vertical velocity $\mathbf{W}(\mathrm{m} / \mathrm{s})$ isosurfaces at $t=300 \mathrm{sec}$ blanked for $y>500 \mathrm{~m}$ for the 3D Robert smooth bubble test on the $30 \mathrm{~m}$ resolution mesh.

area of research. Previous studies [52] shows that the ratio of computational time over communication time is proportional to the order of the WENO finite volume-numerical methods. In other words, the computational time is one order of magnitude greater than the communication time required for each iteration, for WENO3 and higher order schemes. One of the advantages of explicit methods used here is that they can be easily parallelised based on the domain decomposition. The software package used for partitioning uniform unstructured meshes and even hybrid meshes is the METIS software package [26]. The present numerical methods are employed in an unstructured flow solver (UCNS3D) capable of handling hybrid meshes in three-dimensions.

The Robert smooth bubble using the finest resolution, unstructured mesh (210, 212 elements) was utilised as a test problem to assess the scalability of the developed numerical schemes. Although this grid resolution for this sample test case is relatively coarse when compared to the grid resolutions employed nowadays for climate and numerical weather prediction, our primary aim is to demonstrate the dependency of the parallel performance with respect to the numerical schemes implemented in 


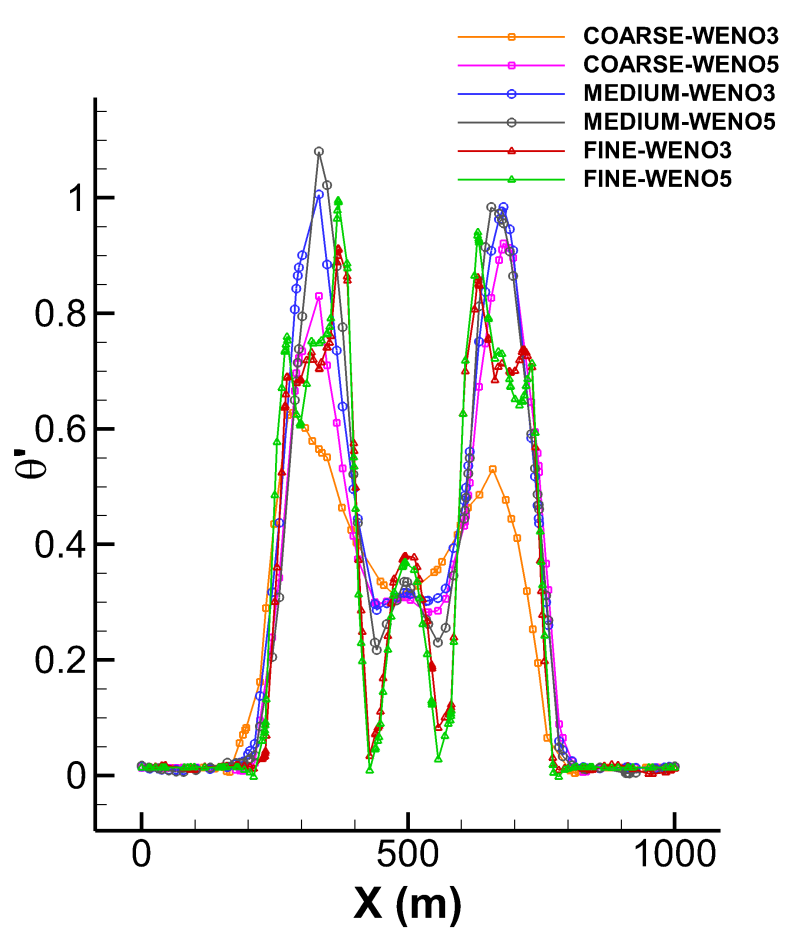

(a) $\theta^{\prime}(K)$

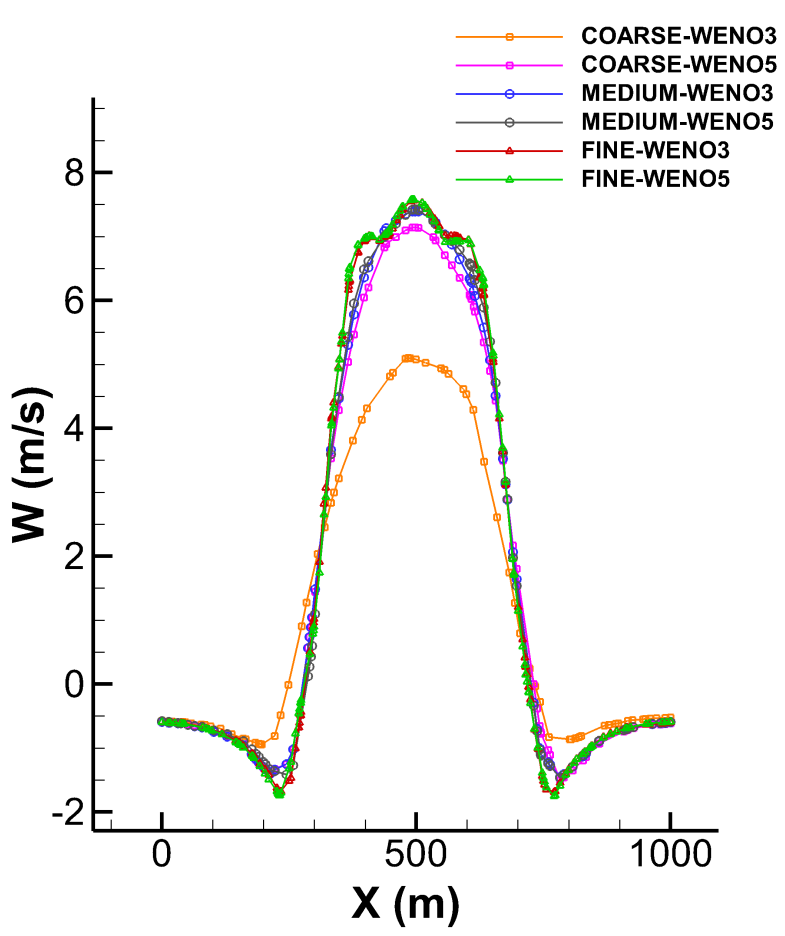

(b) $\mathbf{W}(m / s)$

FIG. 15: Potential temperature perturbation $\theta^{\prime}(K)$ and vertical velocity $\mathbf{W}(\mathrm{m} / \mathrm{s})$ at $t=300 \mathrm{sec}$ $y=500 m$ and $z=964.5 m$ for the Robert smooth bubble test with different schemes depicted represented by different colours (colour online).

the same software framework. The performance tests were conducted at the ARCHER UK National Supercomputing Service at EPCC. The results were obtained using the latest Intel FORTRAN compiler and Intel MPI library.

The time required for each time-step for the WENO3 and WENO5 schemes with respect to the number of processors is illustrated in Fig. 16. The time is normalised with the time required for the WENO3 scheme.

The results obtained confirm that high order methods scale better than lower order methods due to the fact that the ratio of computational time over communication time is greater in the latter case. Using 6144 processors, the parallel efficiency for the different schemes is: WENO3 (67\%) and WENO5 (87\%). It is pointed out that for the WENO5 scheme $92 \%$ of the computational time is spent in the reconstruction process. The exchange of the updated values of the stencil elements takes less than $3 \%$ of the total communication time since the largest communication requirement is the exchange of 


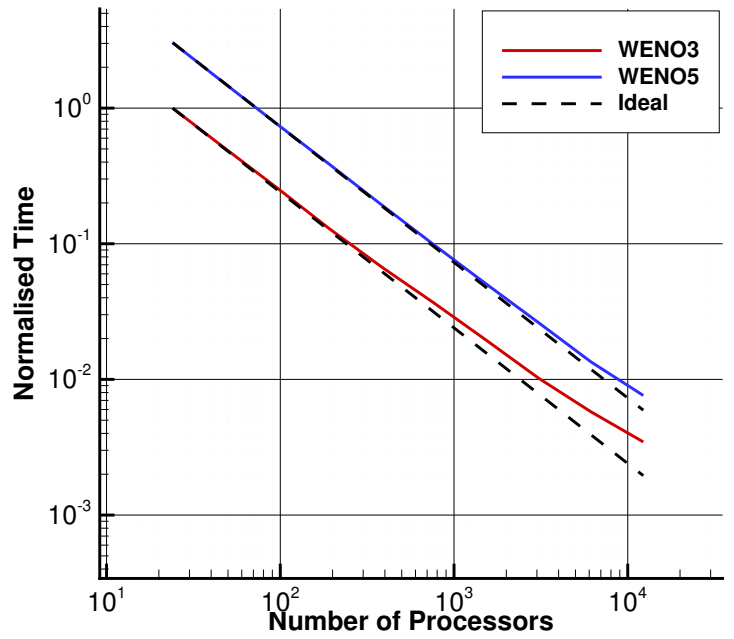

(a) Scalability

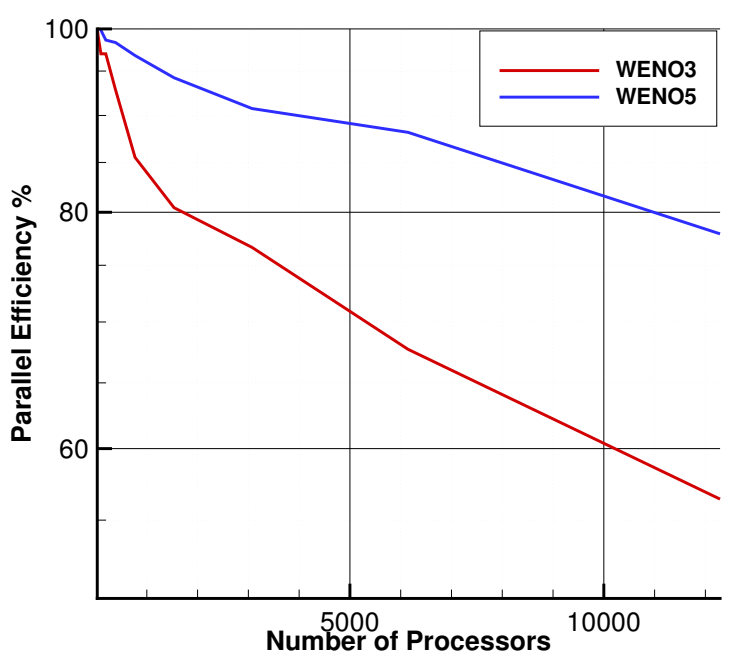

(b) Efficiency

FIG. 16: Scalability and efficiency of WENO3 and WENO5 methods

the reconstructed values for each quadrature point of halo-cells. WENO high-order schemes have a greater computational cost, but they are expected to be superior in accuracy when problems with large time scales are involved such as numerical climate modelling. For numerical climate modelling where projections of the climate for centuries are studied, WENO schemes are expected to have the strength of sufficiently small dissipation errors that will not contaminate the projected solution as a lower-order numerical scheme. Additionally, WENO methods offer better scalability when compared to lowerorder methods.

It has to be noted that when using 6144 processors, each processor holds approximately 35 elements and at 12288 processors, each processor has less than 18 elements which might seem extreme. On the other hand though it might become quite common in the future where exaflop systems will become available and these numbers of processors for such a coarse mesh might become common practice. These results also illustrate that improving the scalability and efficiency of numerical weather prediction models in order to take advantage of massive parallel systems, does not only require the optimisation of the computational software (software engineering), but it also requires re-designing numerical methods in such a way that will benefit from these systems. 


\section{CONCLUSIONS}

This paper presented the implementation of high-order finite-volume schemes for benchmark test cases of stratified atmosphere using the compressible Euler equations on unstructured meshes. The present study discussed the implementation of WENO numerical schemes in this context and assesses their performance in terms of accuracy, robustness, efficiency and scalability. The results demonstrate the aforementioned properties of the schemes in a series of two-dimensional and three-dimensional test cases, and the associated challenges of multidimensional discretisations. What is important to note is that the WENO reconstruction can be utilised as a building-block in a dynamical core that is not limited by the type of meshes, or the formulation of the governing equations. Future work will concern application of the schemes in regional and global atmospheric simulations.

\section{Acknowledgments}

The authors acknowledge the computing time on the UK national high-performance computing service ARCHER that was provided through the UK turbulence consortium in the framework of the EPSRC grant EP/G069581/1.

[1] R. Abgrall. On essentially non-oscillatory schemes on unstructured meshes: analysis and implementation. Journal of Computational Physics, 114(1):45-58, 1994.

[2] N. Ahmad and J. Lindeman. Euler solutions using flux-based wave decomposition. International Journal for Numerical Methods in Fluids, 54:47-72, 2007.

[3] A. F. Antoniadis, D. Drikakis, I. Kokkinakis, P. Tsoutsanis, and Z. Rana. High-order methods for hypersonic shock wave turbulent boundary layer interaction flow. In AIAA, editor, 20th AIAA International Space Planes and Hypersonic Systems and Technologies Conference, number AIAA 2015-3524, Glasgow, Scotland, July 2015.

[4] A. F. Antoniadis, P. Tsoutsanis, and D. Drikakis. Numerical accuracy in rans computations of high-lift multi-element airfoil and aircraft configurations. In AIAA, editor, 53rd AIAA Aerospace Sciences Meeting, number AIAA 2015-0317, Kissimmee, Florida, January 2015. 
[5] S. Brdar, M. Baldauf, A. Dedner, and R. Klofkorn. Comparison of dynamical cores for nwp models: comparison of cosmo and dune. Theoretical and Computational Fluid Dynamics, 27:453-472, 2013.

[6] C. Breviglieri, A.F.S. Maximiliano, E. Basso, and J.L.F. Azevedo. Improved high-order spectral finite volume method implementation for aerodynamic flows. 2009.

[7] B. Cockburn and C.-W. Shu. Runge-kutta discontinuous galerkin methods for convection-dominated problems. Journal of Scientific Computing, 16(3):173-261, 2001.

[8] J.M. Dennis, R.D. Nair, H.M. Tufo, M. Levy, and T. Voran. Development of a scalable global discontinuous galerkin atmospheric model. Int. J. Comp. Sci. Eng.

[9] D. Drikakis, A. F. Antoniadis, P. Tsoutsanis, I. Kokkinakis, and Z. Rana. Azure: An advanced cfd software suite based on high-resolution and high-order methods. In AIAA, editor, 53rd AIAA Aerospace Sciences Meeting, number AIAA 2015-0813, Kissimmee, Florida, January 2015.

[10] M. Dumbser, D.S. Balsara, E.F. Toro, and C.-D. Munz. A unified framework for the construction of onestep finite volume and discontinuous galerkin schemes on unstructured meshes. Journal of Computational Physics, 227(18):8209-8253, 2008.

[11] M. Dumbser, A. Hidalgo, and O. Zanotti. High order space-time adaptive ader-weno finite volume schemes for non-conservative hyperbolic systems. Computer Methods in Applied Mechanics and Engineering, 268:359-387, 2014.

[12] M. Dumbser and M. Kaser. Arbitrary high order non-oscillatory finite volume schemes on unstructured meshes for linear hyperbolic systems. Journal of Computational Physics, 221(2):693-723, 2007.

[13] M. Dumbser, M. Kaser, V.A. Titarev, and E.F. Toro. Quadrature-free non-oscillatory finite volume schemes on unstructured meshes for nonlinear hyperbolic systems. Journal of Computational Physics, 226(1):204243, 2007.

[14] O. Friedrich. Weighted essentially non-oscillatory schemes for the interpolation of mean values on unstructured grids. Journal of Computational Physics, 144(1):194-212, 1998.

[15] F. X. Giraldo, J. F. Kelly, and E. M. Constantinescu. Implicit-explicit formulations of a three-dimensional nonhydrostatic unified model of the atmosphere (numa). SIAM Journal of Scientific Computing, 35:B1162B1194, 2013.

[16] F.X. Giraldo and M. Restelli. A study of spectral element and discontinuous galerkin methods for the navier-stokes equations in nonhydrostatic mesoscale atmospheric modeling: Equation sets and test cases. Journal of Computational Physics, 227(8):3849-3877, 2008. 
[17] F.X. Giraldo, M. Restelli, and M. Louter. Semi-implicit formulations of the navier-stokes equations: Application to nonhydrostatic atmospheric modeling. SIAM Journal on Scientific Computing, 32(6):3394-3425, 2010.

[18] Giraldo, F. Non-hydrostatic Unified Model of the Atmosphere (NUMA), 2015.

[19] C. Ollivier Gooch and M. Van Altena. A high-order-accurate unstructured mesh finite-volume scheme for the advection-diffusion equation. Journal of Computational Physics, 181(2):729-752, 2002.

[20] S. Gottlieb. On high order strong stability preserving runge-kutta and multi step discretizations. Journal of Scientific Computing, 25(112):105-128, 2005.

[21] W.W. Grabowski and T.L. Clark. Cloud-environment interface instablility: rising thermal calculations in two spatial dimensions. Journal of Atmospheric Sciences, 48(1):527-546, 1991.

[22] A. Haselbacher. A weno reconstruction algorithm for unstructured grids based on explicit stencil construction. pages 3369-3378, 2005.

[23] G. Hu, R. Li, and T. Tang. A robust weno type finite volume solver for steady euler equations on unstructured grids. Communications in Computational Physics, 9(3):627-648, 2011.

[24] G. S Jiang and C. W Shu. Efficient implementation of weighted eno schemes. Journal of Computational Physics, 126(1):202-228, 1996.

[25] D. Kalise and I. Lie. Modeling and numerical approximation of a $2.5 \mathrm{~d}$ set of equations for mesoscale atmospheric processes. Journal of Computational Physics, 231:7274-7298, 2012.

[26] G. Karypis and V. Kumar. Multilevel k-way partitioning scheme for irregular graphs. J. Parallel Distrib. Comput., 48:96-129, 1998.

[27] J.F. Kelly, F.X. Giraldo, and G. Jost. Scalability of semi-implicit time integrators for nonhydrostatic galerkin-based atmospheric models on large scale clusters. pages 561-565, 2011.

[28] B. Van Leer. Towards the ultimate conservative difference scheme. ii. monotonicity and conservation combined in a second-order scheme. J. Comput. Phys., 14:361-370, 1974.

[29] B. Van Leer. Towards the ultimate conservative difference scheme. v. a second-order sequel to godunov's method. J. Comput. Phys., 32(1):101-136, 1979.

[30] W.A. Li and Y.X. Ren. Quadrature-free non-oscillation finite volume scheme for solving navier-stokes equations on unstructured grids. volume 1376, pages 639-641, 2011.

[31] S. Marras, M. Moragues, M. Vazquez, O. Jorba, and G. Houzeaux. A variational multiscale stabilized finite element method for the solution of the euler equations of nonhydrostatic stratified flows. Journal of 
Computational Physics, 236(1):380-407, 2013.

[32] S. Marras, M. Nazarov, and F.X. Giraldo. Stabilized high-order galerkin methods based on a parameter-free dynamic sgs model for les. Journal of Computational Physics, 301:77-101, 2015.

[33] Max Planck Institute for Metereology and Deutscher Wetterdienst. ICOsahedral Non-hydrostatic General Circulation Model, 2015.

[34] A. Muller, J. Behrens, F. X. Giraldo, and V. Wirth. Comparison between adaptive and uniform discontinuous galerkin simulations in dry 2d bubble experiments. Journal of Computational Physics, 235:371-393, 2013.

[35] A. Muller, J. Behrens, F.X. Giraldo, and V. Wirth. Comparison between adaptive and uniform discontinuous galerkin simulations in dry 2d bubble experiments. Journal of Computational Physics, 235:371-393, 2013.

[36] National Center for Atmospheric Research. High-Order Methods Modeling Environment, 2015.

[37] National Oceanic Atmospheric Administration. Am3, 2015.

[38] National Oceanic Atmospheric Administration. Nonhydrostatic Icosahedral Model NIM, 2015.

[39] X. Nogueira, L. Cueto-Felgueroso, I. Colominas, F. Navarrina, and M. Casteleiro. A new shock-capturing technique based on moving least squares for higher-order numerical schemes on unstructured grids. Computer Methods in Applied Mechanics and Engineering, 199(37-40):2544-2558, 2010.

[40] M.R. Norman, R.D. Nair, and F.H.M. Semazzi. A low communication and large time step explicit finitevolume solver for non-hydrostatic atmospheric dynamics. Journal of Computational Physics, 230(4):15671584, 2011.

[41] C.F. Ollivier-Gooch. Quasi-eno schemes for unstructured meshes based on unlimited data-dependent leastsquares reconstruction. Journal of Computational Physics, 133(1):6-17, 1997.

[42] A. Robert. Bubble convection experiments with a semi-implicit formulation of the euler equations. Journal of the Atmospheric Sciences, 50(13):1865-1873, 1993.

[43] M. Satoh, T. Matsuno, H. Tomita, H. Miura, T. Nasuno, and S. Iga. Nonhydrostatic icosahedral atmospheric model (nicam) for global cloud resolving simulations. Journal of Computational Physics, 227(7):34863514, 2008.

[44] J. Shi, C. Hu, and C.-W. Shu. A technique of treating negative weights in weno schemes. Journal of Computational Physics, 175(1):108-127, 2002.

[45] C.-W. Shu and S. Osher. Efficient implementation of essentially non-oscillatory shock-capturing schemes. Journal of Computational Physics, 77(2):439-471, 1988.

[46] P. K. Smolarkiewicz, Szmelter J., and A. A. Wyszogrodzki. An unstructured-mesh atmospheric model for 
Journal of Coupled Systems and Multiscale Dynamics • October 2016

nonhydrostatic dynamics. Journal of Computational Physics, 254:184-199, 2013.

[47] P.K. Smolarkiewicz. Multidimensional positive definite advection transport algorithm: An overview. International Journal for Numerical Methods in Fluids, 50(10):1123-1144, 2006.

[48] D. Stanescu and W.G. Habashi. Essentially nonoscillatory euler solutions on unstructured meshes using extrapolation. AIAA Journal, 36(8):1413-1416, 1998.

[49] J.M. Straka, Robert B. Wilhelmson, Louis J. Wicker, John R. Anderson, and Kelvin K. Droegemeier. Numerical solutions of a non-linear density current: a benchmark solution and comparisons. International Journal for Numerical Methods in Fluids, 17(1):1-22, 1993.

[50] V.A. Titarev, P. Tsoutsanis, and D. Drikakis. Weno schemes for mixed-element unstructured meshes. Communications in Computational Physics, 8(3):585-609, 2010.

[51] Eleuterio F. Toro. Riemann Solvers and Numerical Methods for Fluid Dynamics - A Practical Introduction. Berlin: Springer-Verlag, 1997.

[52] P. Tsoutsanis, A.F. Antoniadis, and D. Drikakis. Weno schemes on arbitrary unstructured meshes for laminar, transitional and turbulent flows. Journal of Computational Physics, 256:254-276, 2014.

[53] P. Tsoutsanis, I.W. Kokkinakis, L. Konozsy, D. Drikakis, R.J.R. Williams, and D.L. Youngs. An investigation of the accuracy and efficiency of structured and unstructured, compressible and incompressible methods for the vortex pairing problem. Computer Methods in Applied Mechanics and Engineering, 293:207$231,2015$.

[54] P. Tsoutsanis, V.A. Titarev, and D. Drikakis. Weno schemes on arbitrary mixed-element unstructured meshes in three space dimensions. Journal of Computational Physics, 230(4):1585-1601, 2011.

[55] UK MET Office and ECMWF. Even Newer Dynamics for General atmospheric modelling of the environment (ENDGame), 2015.

[56] A. Uranga, P.-O. Persson, M. Drela, and J. Peraire. Implicit large eddy simulation of transition to turbulence at low reynolds numbers using a discontinuous galerkin method. International Journal for Numerical Methods in Engineering, 87(1-5):232-261, 2011.

[57] Z.J. Wang. Spectral (finite) volume method for conservation laws on unstructured grids. basic formulation. Journal of Computational Physics, 178(1):210-251, 2002.

[58] Z.J. Wang and Y. Liu. Spectral (finite) volume method for conservation laws on unstructured grids. ii. extension to two-dimensional scalar equation. Journal of Computational Physics, 179(2):665-697, 2002.

[59] Z.J. Wang, L. Zhang, and Y. Liu. Spectral (finite) volume method for conservation laws on unstructured 
grids iv: Extension to two-dimensional systems. Journal of Computational Physics, 194(2):716-741, 2004.

[60] W.R. Wolf and J.L.F. Azevedo. High-order eno and weno schemes for unstructured grids. International Journal for Numerical Methods in Fluids, 55(10):917-943, 2007.

[61] Z. Xu, Y. Liu, and C.-W. Shu. Hierarchical reconstruction for discontinuous galerkin methods on unstructured grids with a weno-type linear reconstruction and partial neighboring cells. Journal of Computational Physics, 228(6):2194-2212, 2009.

[62] Z. Xu, Y. Liu, and C.-W. Shu. Hierarchical reconstruction for spectral volume method on unstructured grids. Journal of Computational Physics, 228(16):5787-5802, 2009.

[63] C. Yang and X. C. Cai. A scalable fully implicit compressible euler solver for mesoscale nonhydrostatic simulation of atmospheric flow. SIAM Journal of Scientific Computing, 36:S23-S47, 2014.

[64] L. Yelash, A. Muller, M. Lukacova-Medvid'ova, F.X. Giraldo, and V. Wirth. Adaptive discontinuous evolution galerkin method for dry atmospheric flow. Journal of Computational Physics, 268:106-133, 2014.

[65] G. Zangl, D. Reinert, P. Ripodas, and M. Baldauf. The icon (icosahedral non-hydrostatic) modelling framework of dwd and mpi-m: Description of the non-hydrostatic dynamical core. Quarterly Journal of the Royal Meteorological Society, 2014.

[66] Y. T. Zhang and C. W. Shu. Third order weno scheme on three dimensional tetrahedral meshes. Communications in Computational Physics, 5(2-4):836-848, 2008.

[67] Y. Zhou and Z.J. Wang. Implicit large eddy simulation of transitional flow over a sd7003 wing using high-order spectral difference method. 2010.

[68] J. Zhu, J. Qiu, C.-W. Shu, and M. Dumbser. Runge-kutta discontinuous galerkin method using weno limiters ii: Unstructured meshes. Journal of Computational Physics, 227(9):4330-4353, 2008. 
2016-09-01

\section{A high-order finite-volume method for atmospheric flows on unstructured grids}

\section{Tsoutsanis, Panagiotis}

American Scientific Publishers

Tsoutsanis, Panagiotis; Drikakis, Dimitris, A high-order finite-volume method for atmospheric flows on unstructured grids, Journal of Coupled Systems and Multiscale Dynamics, Volume 4, Issue 3, 2016, pp. 170-186

https://doi.org/10.1166/jcsmd.2016.1104

Downloaded from Cranfield Library Services E-Repository 